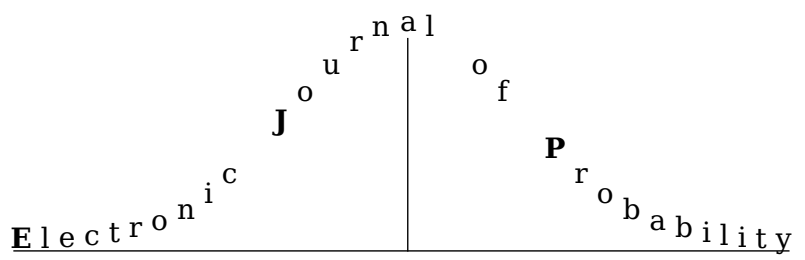

Electron. J. Probab. 26 (2021), article no. 29, 1-29.

ISSN: 1083-6489 https://doi.org/10.1214/20-EJP576

\title{
Support characterization for regular path-dependent stochastic Volterra integral equations
}

\author{
Alexander Kalinin*
}

\begin{abstract}
We consider a stochastic Volterra integral equation with regular path-dependent coefficients and a Brownian motion as integrator in a multidimensional setting. Under an imposed absolute continuity condition, the unique solution is a semimartingale that admits almost surely Hölder continuous paths. Based on functional Itô calculus, we prove that the support of its law in Hölder norms can be described by a flow of mild solutions to ordinary integro-differential equations that are constructed by means of the vertical derivative of the diffusion coefficient.
\end{abstract}

Keywords: support of a measure; path-dependent Volterra process; functional Volterra integral equation; functional Itô calculus; vertical derivative; Hölder space.

MSC2020 subject classifications: 60H20; 28C20; 60G17; 45D05; $45 \mathrm{~J} 05$.

Submitted to EJP on May 25, 2020, final version accepted on December 21, 2020.

Supersedes arXiv: 1908.10786.

\section{Support representations via flows}

The support of the law of a continuous process consists of all continuous paths around any neighborhood the process may stay with positive probability. Determining this class of paths for a diffusion process, viewed as solution to a stochastic differential equation (SDE), establishes a relation between the coefficients of the equation and the law of its solution.

In the pioneering work of Stroock and Varadhan [14], the support of the law of a diffusion process is characterized by an associated flow of classical solutions to ordinary differential equations. While Aida [1] generalizes the time-homogeneous case to a Hilbert space, allowing for an infinite dimension, Gyöngy and Pröhle [9] deal with coefficients that are of affine growth and not necessarily bounded. Moreover, Pakkanen [12] provides sufficient conditions for a stochastic integral to have the full support property.

An extension of the Stroock-Varadhan support theorem to any $\alpha$-Hölder norm, where $\alpha \in(0,1 / 2)$, is given in Ben Arous et al. [4]. The case of time-homogeneous coefficients was independently proven by Millet and Sanz-Solé [11] and later extended to a parabolic

\footnotetext{
${ }^{*}$ Department of Mathematics, LMU Munich, Germany. E-mail: alex.kalinin@mail.de
} 
stochastic partial differential equation (SPDE) in Bally et al. [3]. By using the vertical derivative as functional space derivative and generalizing the approach in [11] with the relevant Girsanov changes of measures, a path-dependent version of the StroockVaradhan support theorem in Hölder norms was recently derived in [7]. The contribution of this article is to extend this support characterization to stochastic Volterra integral equations with regular path-dependent coefficients by providing a flow of mild solutions to ordinary integro-differential equations.

Let $r, T \geq 0$ with $r<T$ and $d, m \in \mathbb{N}$. We work with the separable Banach space $C\left([0, T], \mathbb{R}^{m}\right)$ of all $\mathbb{R}^{m}$-valued continuous paths on $[0, T]$, endowed with the supremum norm given by $\|x\|_{\infty}=\sup _{t \in[0, T]}|x(t)|$, where $|\cdot|$ is used as absolute value function, Euclidean norm or Hilbert-Schmidt norm. Throughout, $\hat{x} \in C\left([0, T], \mathbb{R}^{m}\right)$ and

$$
b:[r, T]^{2} \times C\left([0, T], \mathbb{R}^{m}\right) \rightarrow \mathbb{R}^{m} \text { and } \sigma:[r, T]^{2} \times C\left([0, T], \mathbb{R}^{m}\right) \rightarrow \mathbb{R}^{m \times d}
$$

are two product measurable maps that are non-anticipative in the sense that they satisfy $b(t, s, x)=b\left(t, s, x^{s}\right)$ and $\sigma(t, s, x)=\sigma\left(t, s, x^{s}\right)$ for all $s, t \in[r, T]$ with $s \leq t$ and each $x \in C\left([0, T], \mathbb{R}^{m}\right)$, where $x^{s}$ denotes the path $x$ stopped at time $s$.

On a filtered probability space $\left(\Omega, \mathscr{F},\left(\mathscr{F}_{t}\right)_{t \in[0, T]}, P\right)$ that satisfies the usual conditions and which allows for a standard $d$-dimensional $\left(\mathscr{F}_{t}\right)_{t \in[0, T]}$-Brownian motion $W$, we consider the following path-dependent stochastic Volterra integral equation:

$$
X_{t}=X_{r}+\int_{r}^{t} b(t, s, X) d s+\int_{r}^{t} \sigma(t, s, X) d W_{s} \text { a.s. }
$$

for $t \in[r, T]$ with initial condition $X_{q}=\hat{x}(q)$ for $q \in[0, r]$ a.s. An absolute continuity and affine growth condition on the coefficients $b$ and $\sigma$ ensures that any solution to (1.1) is a semimartingale with delayed Hölder continuous trajectories.

In fact, for each $\alpha \in(0,1]$ let $C_{r}^{\alpha}\left([0, T], \mathbb{R}^{m}\right)$ represent the non-separable Banach space of all $x \in C\left([0, T], \mathbb{R}^{m}\right)$ that are $\alpha$-Hölder continuous on $[r, T]$, endowed with the delayed $\alpha$-Hölder norm given by

$$
\|x\|_{\alpha, r}:=\left\|x^{r}\right\|_{\infty}+\sup _{s, t \in[r, T]: s \neq t} \frac{|x(s)-x(t)|}{|s-t|^{\alpha}} .
$$

By convention, we set $C_{r}^{0}\left([0, T], \mathbb{R}^{m}\right):=C\left([0, T], \mathbb{R}^{m}\right)$ and $\|\cdot\|_{0, r}:=\|\cdot\|_{\infty}$. Then, under the conditions stated below, there is a unique strong solution to (1.1) whose sample paths belong a.s. to the delayed Hölder space $C_{r}^{\alpha}\left([0, T], \mathbb{R}^{m}\right)$ for any $\alpha \in(0,1 / 2)$.

For $p \geq 1$ consider the separable Banach space $W_{r}^{1, p}\left([0, T], \mathbb{R}^{m}\right)$ of all $x \in C\left([0, T], \mathbb{R}^{m}\right)$ that are absolutely continuous on $[r, T]$ with a $p$-fold Lebesgue-integrable weak derivative $\dot{x}$, equipped with the delayed Sobolev $L^{p}$-norm defined by

$$
\|x\|_{1, p, r}:=\left\|x^{r}\right\|_{\infty}+\left(\int_{r}^{t}|\dot{x}(s)|^{p} d s\right)^{\frac{1}{p}} .
$$

Then it holds that $W_{r}^{1, p}\left([0, T], \mathbb{R}^{m}\right) \subsetneq C_{r}^{1 / q}\left([0, T], \mathbb{R}^{m}\right)$ and $\|x\|_{1 / q, r} \leq\|x\|_{1, p, r}$ for all $x \in W_{r}^{1, p}\left([0, T], \mathbb{R}^{m}\right)$ whenever $p>1$ and $q$ is its dual exponent. By allowing infinite values, we extend the definitions of $\|\cdot\|_{\infty}$ and $\|\cdot\|_{\alpha, r}$ at (1.2) to each path $x:[0, T] \rightarrow \mathbb{R}^{m}$ and the definition of $\|\cdot\|_{1, p, r}$ at (1.3) to every $x \in W_{r}^{1,1}\left([0, T], \mathbb{R}^{m}\right)$.

Based on the non-separable Banach space $D\left([0, T], \mathbb{R}^{m}\right)$ of all $\mathbb{R}^{m}$-valued càdlàg paths on $[0, T]$, endowed with the supremum norm $\|\cdot\|_{\infty}$, we use the following pseudometric on $[r, T] \times D\left([0, T], \mathbb{R}^{m}\right)$ given by

$$
d_{\infty}((t, x),(s, y)):=|t-s|^{\frac{1}{2}}+\left\|x^{t}-y^{s}\right\|_{\infty} .
$$


Then a functional on this Cartesian product that is $d_{\infty}$-continuous is also non-anticipative and Lipschitz continuity relative to $d_{\infty}$ merely requires $1 / 2$-Hölder continuity in the time variable.

Let us now state the conditions under which the support theorem holds. By referring to horizontal and vertical differentiability of non-anticipative functionals from [5, 8], we in particular require that certain time and path space components of $\sigma$ are of class $\mathbb{C}^{1,2}$, a property to be recalled in Section 2.1. In this context, let $\partial_{s}$ be the horizontal, $\partial_{x}$ the vertical and $\partial_{x x}$ the second-order vertical differential operator.

To have a simple notation if these first- and second-order space derivatives appear, we set $\|y\|:=\left(\sum_{k=1}^{m} \sum_{l=1}^{d}\left|y_{k, l}\right|^{2}\right)^{1 / 2}$ if $y \in\left(\mathbb{R}^{1 \times m}\right)^{m \times d}$ or $y \in\left(\mathbb{R}^{m \times m}\right)^{m \times d}$. Further, let $\mathbb{I}_{d}$ be the identity matrix in $\mathbb{R}^{d \times d}$ and $A^{\prime}$ denote the transpose of a matrix $A \in \mathbb{R}^{m \times d}$.

(C.1) The map $[r, t) \times C\left([0, T], \mathbb{R}^{m}\right) \rightarrow \mathbb{R}^{m \times d},(s, x) \mapsto \sigma(t, s, x)$ is of class $\mathbb{C}^{1,2}$ for each $t \in(r, T]$, the maps

$$
b(\cdot, s, x) \text { and } \sigma(\cdot, s, x)
$$

are absolutely continuous on $[s, T]$ and $\partial_{x} \sigma(\cdot, s, x)$ is absolutely continuous on $(s, T]$ for any $(s, x) \in[r, T) \times C\left([0, T], \mathbb{R}^{m}\right)$.

(C.2) The maps $\sigma, \partial_{x} \sigma$ and its weak time derivatives $\partial_{t} \sigma, \partial_{t} \partial_{x} \sigma$ are bounded. Further, there are $c, \eta \geq 0$ and $\kappa \in[0,1)$ such that

$$
\begin{aligned}
|b(s, s, x)|+\left|\partial_{t} b(t, s, x)\right| & \leq c\left(1+\|x\|_{\infty}^{\kappa}\right) \\
\text { and } \quad\left|\partial_{s} \sigma(t, s, x)\right|+\left\|\partial_{x x} \sigma(t, s, x)\right\| & \leq c\left(1+\|x\|_{\infty}^{\eta}\right)
\end{aligned}
$$

for all $s, t \in[r, T)$ with $s<t$ and each $x \in C\left([0, T], \mathbb{R}^{m}\right)$.

(C.3) There is $\lambda \geq 0$ satisfying $|b(s, s, x)-b(s, s, y)|+\left|\partial_{t} b(t, s, x)-\partial_{t} b(t, s, y)\right| \leq \lambda\|x-y\|_{\infty}$ and

$$
\begin{aligned}
|\sigma(u, t, x)-\sigma(u, s, y)| & +\left|\partial_{u} \sigma(u, t, x)-\partial_{u} \sigma(u, s, y)\right| \\
& +\left\|\partial_{x} \sigma(u, t, x)-\partial_{x} \sigma(u, s, y)\right\| \leq \lambda d_{\infty}((t, x),(s, y))
\end{aligned}
$$

for any $s, t, u \in[r, T)$ with $s<t<u$ and every $x, y \in C\left([0, T], \mathbb{R}^{m}\right)$.

Under the assumption that $\sigma(t, \cdot, \cdot)$ is of class $\mathbb{C}^{1,2}$ on $[r, t) \times C\left([0, T], \mathbb{R}^{m}\right)$ for each $t \in(r, T]$, we may introduce the map $\rho:[r, T]^{2} \times C\left([0, T], \mathbb{R}^{m}\right) \rightarrow \mathbb{R}^{m}$, which serves as correction term, coordinatewise by

$$
\rho_{k}(t, s, x)=\sum_{l=1}^{d} \partial_{x} \sigma_{k, l}(t, s, x) \sigma(s, s, x) e_{l},
$$

if $s<t$, and $\rho_{k}(t, s, x):=0$, otherwise. Here, $\left\{e_{1}, \ldots, e_{d}\right\}$ stands for the standard basis of $\mathbb{R}^{d}$ and $[r, t) \times C\left([0, T], \mathbb{R}^{m}\right) \rightarrow \mathbb{R}^{1 \times m},(s, x) \mapsto \partial_{x} \sigma_{k, l}(t, s, x)$ is the vertical derivative of the $(k, l)$-entry of the map $[r, t) \times C\left([0, T], \mathbb{R}^{m}\right) \rightarrow \mathbb{R}^{m \times d},(s, x) \mapsto \sigma(t, s, x)$ for each $t \in(r, T]$, every $k \in\{1, \ldots, m\}$ and any $l \in\{1, \ldots, d\}$.

Finally, to describe the support of the unique strong solution to (1.1) by a flow, we study the following path-dependent Volterra integral equation associated to any $h \in W_{r}^{1, p}\left([0, T], \mathbb{R}^{d}\right)$ with $p \geq 2$. Namely,

$$
x_{h}(t)=x_{h}(r)+\int_{r}^{t}(b-(1 / 2) \rho)\left(t, s, x_{h}\right) d s+\int_{r}^{t} \sigma\left(t, s, x_{h}\right) d h(s)
$$

for $t \in[r, T]$. By adding $\hat{x}$ as initial condition, the solution $x_{h}$ lies in the delayed Sobolev space $W_{r}^{1, p}\left([0, T], \mathbb{R}^{m}\right)$, since it can also be viewed as a mild solution to an associated ordinary integro-differential equation, as concisely justified in Section 2.2. 
Lemma 1.1. Let (C.1)-(C.3) be valid.

(i) Pathwise uniqueness holds for (1.1) and there is a unique strong solution $X$ such that $X^{r}=\hat{x}^{r}$ a.s. Further, $X$ is a semimartingale and $E\left[\|X\|_{\alpha, r}^{p}\right]<\infty$ for any $\alpha \in[0,1 / 2)$ and all $p \geq 2$.

(ii) For any $p \geq 2$ and each $h \in W_{r}^{1, p}\left([0, T], \mathbb{R}^{d}\right)$, there is a unique solution $x_{h}$ to (1.5) satisfying $x_{h}^{r}=\hat{x}^{r}$ and we have $x_{h} \in W_{r}^{1, p}\left([0, T], \mathbb{R}^{m}\right)$. Moreover, the flow map

$$
W_{r}^{1, p}\left([0, T], \mathbb{R}^{d}\right) \rightarrow W_{r}^{1, p}\left([0, T], \mathbb{R}^{m}\right), \quad h \mapsto x_{h}
$$

is Lipschitz continuous on bounded sets.

Having clarified matters of uniqueness, existence and regularity, let us now consider the main result of this paper. Namely, a support characterization for solutions to (1.1) in delayed Hölder norms.

Theorem 1.2. Let (C.1)-(C.3) hold, $\alpha \in[0,1 / 2)$ and $p \geq 2$. Then the support of the image measure of the unique strong solution $X$ to (1.1) in $C_{r}^{\alpha}\left([0, T], \mathbb{R}^{m}\right)$ is the closure of the set of all solutions $x_{h}$ to (1.5), where $h \in W_{r}^{1, p}\left([0, T], \mathbb{R}^{d}\right)$. That is,

$$
\operatorname{supp}\left(P \circ X^{-1}\right)=\overline{\left\{x_{h} \mid h \in W_{r}^{1, p}\left([0, T], \mathbb{R}^{d}\right)\right\}} \text { in } C_{r}^{\alpha}\left([0, T], \mathbb{R}^{m}\right) .
$$

Example 1.3. Suppose there are four product measurable maps $k_{b}, k_{\sigma}:[r, T]^{2} \rightarrow \mathbb{R}$, $\bar{b}:[r, T] \times C\left([0, T], \mathbb{R}^{m}\right) \rightarrow \mathbb{R}^{m}$ and $\bar{\sigma}:[r, T] \times C\left([0, T], \mathbb{R}^{m}\right) \rightarrow \mathbb{R}^{m \times d}$ such that

$$
b(t, s, x)=k_{b}(t, s) \bar{b}(s, x) \text { and } \sigma(t, s, x)=k_{\sigma}(t, s) \bar{\sigma}(s, x)
$$

for all $s, t \in[r, T]$ and any $x \in C\left([0, T], \mathbb{R}^{m}\right)$ and let the following three conditions hold:

(1) The functions $k_{b}(\cdot, s)$ and $k_{\sigma}(\cdot, s)$ are absolutely continuous on $[s, T]$ for all $s \in[r, T)$ and $k_{\sigma}(t, \cdot)$ is continuously differentiable on $[r, t)$ for any $t \in(r, T]$. Further, $k_{b}, k_{\sigma}$, $\partial_{t} k_{b}, \partial_{t} k_{\sigma}$ and $\partial_{s} k_{\sigma}$ are bounded.

(2) The map $\bar{\sigma}$ is of class $\mathbb{C}^{1,2}$ on $[r, T) \times C\left([0, T], \mathbb{R}^{m}\right)$ and together with its vertical derivative $\partial_{x} \bar{\sigma}$ it is bounded and $d_{\infty}$-Lipschitz continuous.

(3) There are $c, \eta, \lambda \geq 0$ and $\kappa \in[0,1)$ so that $|\bar{b}(s, x)| \leq c\left(1+\|x\|_{\infty}^{\kappa}\right),|\bar{b}(s, x)-\bar{b}(s, y)|$ $\leq \lambda\|x-y\|_{\infty},\left|k_{\sigma}(u, t)-k_{\sigma}(u, s)\right|+\left|\partial_{u} k_{\sigma}(u, t)-\partial_{u} k_{\sigma}(u, s)\right| \leq \lambda|s-t|^{1 / 2}$ and

$$
\left|\partial_{s} \bar{\sigma}(s, x)\right|+\left\|\partial_{x x} \bar{\sigma}(s, x)\right\| \leq c\left(1+\|x\|_{\infty}^{\eta}\right)
$$

for all $s, t, u \in[r, T)$ with $s<t<u$ and any $x, y \in C\left([0, T], \mathbb{R}^{m}\right)$.

Then Theorem 1.2 applies and for $k_{b}=k_{\sigma}=1$ it reduces to the support theorem in [7] with the same regularity conditions. Moreover, we could also take $k_{b}(t, s)=(t-s)^{\beta_{b}}$ and $k_{\sigma}(t, s)=(t-s)^{\beta_{\sigma}-\frac{1}{2}}$ for any $s, t \in[r, T]$ with $s \leq t$, where $\beta_{b} \geq 1$ and $\beta_{\sigma} \geq 2$.

The structure of this paper is determined by the proof of the support theorem and can be comprised as follows. Section 2 provides supplementary material and a convergence result in Hölder norms that yields Theorem 1.2 as a corollary. In detail, Section 2.1 gives a concise overview of horizontal and vertical differentiability of non-anticipative functionals. Section 2.2 relates the Volterra integral equation (1.5) to an ordinary integro-differential equation and shows that solutions to (1.1) are semimartingales by using a stochastic Fubini theorem. In Section 2.3 we consider the approach to prove the support theorem by introducing a more general setting and stating Theorem 2.3, the before mentioned convergence result. 
Section 3 derives relevant estimates to infer convergence in Hölder norms in moments. To be precise, Section 3.1 gives a sufficient condition for a sequence of processes to converge in this sense by exploiting an explicit Kolmogorov-Chentsov estimate. In Section 3.2 we introduce the relevant notations in the context of sequences of partitions and recall a couple of auxiliary moment estimates from [7, 10]. The purpose of Section 3.3 is to deduce moment estimates for deterministic and stochastic Volterra integrals, generalizing the bounds from [7][Lemmas 20, 21 and Proposition 22].

Section 4 is devoted to a variety of specific moment estimates and decompositions, preparing the proof of Theorem 2.3. At first, Section 4.1 derives bounds for solutions to stochastic Volterra integral equations and gives two main decompositions, Proposition 4.3 and equation (4.7). Section 4.2 handles the first two remainders appearing in (4.7). While the second can be directly estimated, the first relies on the functional Itô formula from [6]. Section 4.3 intends to bound the third remainder in second moment, requiring another extensive decomposition. In Section 5 we prove the convergence result and the support representation, including assertions on uniqueness, existence and regularity.

\section{Preparations and a convergence result in second moment}

\subsection{Differential calculus for non-anticipative functionals}

We recall and discuss horizontal and vertical differentiability, as introduced in $[5,8]$. To this end, let $t \in(r, T]$ and $G$ be a non-anticipative functional on $[r, t) \times D\left([0, T], \mathbb{R}^{m}\right)$ that is considered at a point $(s, x)$ of its domain:

(i) $G$ is horizontally differentiable at $(s, x)$ if the function $[0, t-s) \rightarrow \mathbb{R}, h \mapsto G\left(s+h, x^{s}\right)$ is differentiable at 0 . If this is the case, then $\partial_{s} G(s, x)$ denotes its derivative there.

(ii) $G$ is vertically differentiable at $(s, x)$ if the function $\mathbb{R}^{m} \rightarrow \mathbb{R}, h \mapsto G\left(s, x+h \mathbb{1}_{[s, T]}\right)$ is differentiable at 0 . In this case, its derivative there is denoted by $\partial_{x} G(s, x)$.

(iii) $G$ is partially vertically differentiable at $(s, x)$ if for any $k \in\{1, \ldots, m\}$ the function $\mathbb{R} \rightarrow \mathbb{R}, h \mapsto G\left(s, x+h \bar{e}_{k} \mathbb{1}_{[s, T]}\right)$ is differentiable at 0 , where $\left\{\bar{e}_{1}, \ldots, \bar{e}_{m}\right\}$ is the standard basis of $\mathbb{R}^{m}$. In this case, $\partial_{x_{k}} G(s, x)$ represents its derivative there.

So, $G$ is horizontally, vertically or partially vertically differentiable if it satisfies the respective property at any point of its domain. We observe that vertical differentiability entails partial vertical differentiability and $\partial_{x} G=\left(\partial_{x_{1}} G, \ldots, \partial_{x_{m}} G\right)$.

We say that $G$ is twice vertically differentiable if it is vertically differentiable and the same is true for $\partial_{x} G$. Then we set $\partial_{x x} G:=\partial_{x}\left(\partial_{x} G\right)$ and $\partial_{x_{k} x_{l}} G:=\partial_{x_{k}}\left(\partial_{x_{l}} G\right)$ for any $k, l \in\{1, \ldots, m\}$. If in addition $\partial_{x x} G$ is $d_{\infty}$-continuous, then

$$
\partial_{x_{k} x_{l}} G=\partial_{x_{l} x_{k}} G \text { for all } k, l \in\{1, \ldots, m\},
$$

by Schwarz's lemma, showing that $\partial_{x x} G$ is symmetric. Moreover, we call $G$ of class $\mathbb{C}^{1,2}$ if it is once horizontally and twice vertically differentiable such that $G, \partial_{s} G, \partial_{x} G$ and $\partial_{x x} G$ are bounded on bounded sets and $d_{\infty}$-continuous.

Clearly, horizontal differentiability applies to functionals on $[r, t) \times C\left([0, T], \mathbb{R}^{m}\right)$ as well by considering continuous paths only. Vertical differentiability, however, requires the evaluation along càdlàg paths. So, a functional $F$ on $[r, t) \times C\left([0, T], \mathbb{R}^{m}\right)$ is of class $\mathbb{C}^{1,2}$ if it possesses a non-anticipative extension $G:[r, t) \times D\left([0, T], \mathbb{R}^{m}\right) \rightarrow \mathbb{R}$ that satisfies this property. Then the restricted derivatives

$$
\partial_{x} F:=\partial_{x} G \quad \text { and } \quad \partial_{x x} F:=\partial_{x x} G \text { on }[r, t) \times C\left([0, T], \mathbb{R}^{m}\right)
$$

are well-defined, by Theorems 5.4.1 and 5.4.2 in [2]. That is, they do not depend on the choice of the extension $G$. By combining these considerations with an absolute continuity 
condition, which ensures that only semimartingales appear, we can use the functional Itô formula from [6] to prove Proposition 4.4, a key component when deriving (1.6).

Examples 2.1. (i) We suppose that $\alpha \in(0,1], k \in \mathbb{N}$ and $\varphi:[r, t) \times\left(\mathbb{R}^{m}\right)^{k} \rightarrow \mathbb{R}^{d}$ is $\alpha$-Hölder continuous. For $t_{1}, \ldots, t_{k} \in[0, T]$ the $\mathbb{R}^{d}$-valued non-anticipative map $G$ on $[r, t) \times D\left([0, T], \mathbb{R}^{m}\right)$ given by

$$
G(s, x):=\varphi\left(s, x\left(s \wedge t_{1}\right), \ldots, x\left(s \wedge t_{k}\right)\right)
$$

is bounded on bounded sets and $\alpha$-Hölder continuous relative to $d_{\infty}$. If $\varphi\left(\cdot, \bar{x}_{1}, \ldots, \bar{x}_{k}\right)$ and the map $\left(\mathbb{R}^{m}\right)^{k} \rightarrow \mathbb{R}^{d},\left(\bar{y}_{1}, \ldots, \bar{y}_{k}\right) \mapsto \varphi\left(s, \bar{y}_{1}, \ldots, \bar{y}_{k}\right)$ are differentiable for each $s \in[r, t)$ and all $\bar{x}_{1}, \ldots, \bar{x}_{k} \in \mathbb{R}^{m}$, then $G$ is horizontally and vertically differentiable with $\partial_{s} G(s, x)=\left(\partial_{+} \varphi / \partial s\right)\left(s, x\left(s \wedge t_{1}\right), \ldots, x\left(s \wedge t_{k}\right)\right)$ and

$$
\partial_{x} G(s, x)=\sum_{j=1}^{k} D_{\bar{x}_{j}} \varphi\left(s, x\left(s \wedge t_{1}\right), \ldots, x\left(s \wedge t_{k}\right)\right) \mathbb{1}_{\left[r, t_{j}\right]}(s)
$$

for any $(s, x) \in[r, t) \times D\left([0, T], \mathbb{R}^{m}\right)$, where $\partial_{+} \varphi / \partial s$ is the right-hand time derivative of $\varphi$ and $D_{\bar{x}_{j}} \varphi$ denotes the derivative of $\varphi$ with respect to the $j$-th space variable $\bar{x}_{j} \in \mathbb{R}^{m}$ for each $j \in\{1, \ldots, k\}$.

While $\partial_{x} G$ may fail to be $d_{\infty}$-continuous if $t_{j} \in[r, t)$ for some $j \in\{1, \ldots, k\}$, we see that $G$ is of class $\mathbb{C}^{1,2}$ as soon as $\varphi$ is of class $C^{1,2}$ in the usual sense and $t_{j} \geq t$ for all $j \in\{1, \ldots, k\}$.

(ii) Let $\alpha \in(0,1], K:[0, t) \rightarrow \mathbb{R}$ be continuously differentiable and $\varphi$ be an $\mathbb{R}^{m \times d}$-valued Borel measurable bounded map on $[0, t) \times D\left([0, T], \mathbb{R}^{m}\right)$ that is $\alpha$-Hölder continuous in $x \in D\left([0, T], \mathbb{R}^{m}\right)$, uniformly in $s \in[0, t)$. Then the non-anticipative kernel integral map $G:[r, t) \times D\left([0, T], \mathbb{R}^{m}\right) \rightarrow \mathbb{R}^{m \times d}$ defined by

$$
G(s, x):=\int_{0}^{s} K(s-u) \varphi\left(u, x^{u}\right) d u
$$

is bounded and $\alpha$-Hölder continuous relative to $d_{\infty}$. In addition, if $\varphi$ is $d_{\infty}$-continuous, then $G$ is of class $\mathbb{C}^{1,2}$, since $\partial_{s} G(s, x)=K(0) \varphi(s, x)+\int_{0}^{s} \dot{K}(s-u) \varphi(u, x) d u$ for each $(s, x) \in[r, t) \times D\left([0, T], \mathbb{R}^{m}\right)$ and $\partial_{x} G=0$.

\subsection{Ordinary integro-differential equations and semimartingales}

By utilizing an absolute continuity condition, we directly connect the Volterra integral equation (1.5) to an ordinary integro-differential equation and check that any solution to (1.1) solves a stochastic differential equation, ensuring that it is a semimartingale.

Let us first briefly analyze (1.5) for $h \in W_{r}^{1,1}\left([0, T], \mathbb{R}^{d}\right)$, under the hypothesis that $\sigma(t, \cdot, \cdot)$ is of class $\mathbb{C}^{1,2}$ on $[r, t) \times C\left([0, T], \mathbb{R}^{m}\right)$ for each $t \in(r, T]$. A solution to (1.5) is a path $x \in C\left([0, T], \mathbb{R}^{m}\right)$ such that

$$
\begin{aligned}
& \int_{r}^{t}|(b-(1 / 2) \rho)(t, s, x)|+|\sigma(t, s, x)||\dot{h}(s)| d s \text { and } \\
& x(t)=x(r)+\int_{r}^{t}(b-(1 / 2) \rho)(t, s, x) d s+\int_{r}^{t} \sigma(t, s, x) d h(s)
\end{aligned}
$$

for any $t \in[r, T]$, since the variation of $h$ on $[r, s]$ is given by $\int_{r}^{s}|\dot{h}(u)| d u$ for all $s \in[r, t]$. If we now assume that (C.1)-(C.3) are valid, then the $d_{\infty}$-Lipschitz continuity of the map $[r, t) \times C\left([0, T], \mathbb{R}^{m}\right) \rightarrow \mathbb{R}^{1 \times m},(s, x) \mapsto \partial_{x} \sigma_{k, l}(t, s, x)$ entails that it admits a unique continuous extension to $[r, t] \times C\left([0, T], \mathbb{R}^{m}\right)$ for any $t \in(r, T]$, each $k \in\{1, \ldots, m\}$ and every $l \in\{1, \ldots, d\}$. 
In this case, we may define $\bar{\rho}:[r, T]^{2} \times C\left([0, T], \mathbb{R}^{m}\right) \rightarrow \mathbb{R}^{m}$ coordinatewise by letting $\bar{\rho}_{k}(t, s, x)$ agree with the right-hand side in (1.4), if $r<t$ and $s \leq t$, and setting $\bar{\rho}(t, s, x):=0$, otherwise. Then Fubini's theorem entails for any $x \in C\left([0, T], \mathbb{R}^{m}\right)$ that

$$
\begin{aligned}
& \int_{r}^{t}(b-(1 / 2) \rho)(t, s, x) d s+\int_{r}^{t} \sigma(t, s, x) d h(s) \\
& =\int_{r}^{t}(b-(1 / 2) \bar{\rho}+\sigma \dot{h})(s, s, x)+\int_{r}^{s} \partial_{s}(b-(1 / 2) \bar{\rho}+\sigma \dot{h})(s, u, x) d u d s
\end{aligned}
$$

for every $t \in[r, T]$. Consequently, the path $x$ solves (1.5) if and only if it is a mild solution to the path-dependent ordinary integro-differential equation

$$
\dot{x}(t)=(b-(1 / 2) \bar{\rho}+\sigma \dot{h})(t, t, x)+\int_{r}^{t} \partial_{t}(b-(1 / 2) \bar{\rho}+\sigma \dot{h})(t, s, x) d s
$$

for $t \in[r, T]$. Since all appearing maps are integrable, this means that the increment $x(t)-x(r)$ agrees with (2.1) for any $t \in[r, T]$. Let us now turn to the stochastic Volterra integral equation (1.1), without imposing any conditions for the moment.

Thus, we let $\mathscr{C}\left([0, T], \mathbb{R}^{m}\right)$ denote the completely pseudometrizable topological space of all $\left(\mathscr{F}_{t}\right)_{t \in[0, T]}$-adapted continuous processes $X:[0, T] \times \Omega \rightarrow \mathbb{R}^{m}$ and recall that a solution to (1.1) is a process $X \in \mathscr{C}\left([0, T], \mathbb{R}^{m}\right)$ such that

$$
\begin{aligned}
& \int_{r}^{t}|b(t, s, X)|+|\sigma(t, s, X)|^{2} d s<\infty \quad \text { a.s. and } \\
& X_{t}=X_{r}+\int_{r}^{t} b(t, s, X) d s+\int_{r}^{t} \sigma(t, s, X) d W_{s} \quad \text { a.s. for all } t \in[r, T] .
\end{aligned}
$$

For a process $\xi \in \mathscr{C}\left([0, T], \mathbb{R}^{m}\right)$ let $\left(\xi_{\xi} \mathscr{E}_{t}^{0}\right)_{t \in[0, T]}$ be the natural filtration of the adapted continuous process $[0, T] \times \Omega \rightarrow \mathbb{R}^{m} \times \mathbb{R}^{d},(t, \omega) \mapsto\left(\xi_{t}^{r}, W_{r \vee t}-W_{r}\right)(\omega)$. Put differently, $\xi_{\mathscr{E}_{t}}^{0}=\sigma\left(\xi_{q}: q \in[0, t]\right)$ for $t \in[0, r]$ and

$$
\xi_{\mathscr{E}}^{\mathscr{E}_{t}^{0}}=\xi_{\mathscr{E}_{r}^{0}}^{0} \vee \sigma\left(W_{s}-W_{r}: s \in[r, t]\right) \text { for } t \in(r, T] .
$$

In particular, $\xi \mathscr{E}_{t}^{0}=\sigma\left(\xi_{0}\right) \vee \sigma\left(W_{s}: s \in[0, t]\right)$ for all $t \in[0, T]$ if there is no delay. Then a solution $X$ to (1.1) satisfying $X^{r}=\xi^{r}$ a.s. is called strong if it is adapted to the right-continuous filtration of the augmented filtration of $\left(\xi_{\xi} \mathscr{E}_{t}^{0}\right)_{t \in[0, T]}$.

Finally, suppose that (C.1) and (C.2) hold. Then it follows from Fubini's theorem for stochastic integrals, stated in [15] for instance, that any $X \in \mathscr{C}\left([0, T], \mathbb{R}^{m}\right)$ satisfies

$$
\int_{r}^{t} b(t, s, X) d s+\int_{r}^{t} \sigma(t, s, X) d W_{s}=\int_{r}^{t} B_{s}(X) d s+\int_{r}^{t} \sigma(s, s, X) d W_{s}
$$

a.s. for any $t \in[r, T]$, where the map $B:[r, T] \times \Omega \times \mathscr{C}\left([0, T], \mathbb{R}^{m}\right) \rightarrow \mathbb{R}^{m}$, which depends on whole processes rather than trajectories and is such that $B(Y)$ is progressively measurable for any $Y \in \mathscr{C}\left([0, T], \mathbb{R}^{m}\right)$, is given by

$$
B_{s}(Y)=b(s, s, Y)+\int_{r}^{s} \partial_{s} b(s, u, Y) d u+\int_{r}^{s} \partial_{s} \sigma(s, u, Y) d W_{u}
$$

for every $s \in[r, T]$ a.s. This shows that $X$ solves (1.1) if and only if it is a solution to the path-dependent stochastic differential equation

$$
X_{t}=B_{t}(X) d t+\sigma(t, t, X) d W_{t} \text { for } t \in[r, T] .
$$

Moreover, its restriction to $[r, T] \times \Omega$ is automatically a semimartingale in this case. 


\subsection{Approach to the main result in a general setting}

After these preliminary considerations, we proceed as follows to establish the support theorem. For any $n \in \mathbb{N}$ let $\mathbb{T}_{n}$ be a partition of $[r, T]$ of the form $\mathbb{T}_{n}=\left\{t_{0, n}, \ldots, t_{k_{n}, n}\right\}$ with $k_{n} \in \mathbb{N}$ and $t_{0, n}, \ldots, t_{k_{n}, n} \in[r, T]$ such that $r=t_{0, n}<\cdots<t_{k_{n}, n}=T$ and whose mesh $\max _{i \in\left\{0, \ldots, k_{n}-1\right\}}\left(t_{i+1, n}-t_{i, n}\right)$ is denoted by $\left|\mathbb{T}_{n}\right|$. We suppose that $\lim _{n \uparrow \infty}\left|\mathbb{T}_{n}\right|=0$ and that there is $c_{\mathbb{T}} \geq 1$ such that

$$
\left|\mathbb{T}_{n}\right| \leq c_{\mathbb{T}} \min _{i \in\left\{0, \ldots, k_{n}-1\right\}}\left(t_{i+1, n}-t_{i, n}\right) \text { for all } n \in \mathbb{N} .
$$

For the estimation of one term in Proposition 4.4, when the functional Itô formula is applied, we also require the following additional condition:

(C.4) There is $\bar{c}_{\mathbb{T}}>0$ such that $k_{n}\left|\mathbb{T}_{n}\right| \leq \bar{c}_{\mathbb{T}}$ for each $n \in \mathbb{N}$.

However, unless explicitly stated, we shall not impose this condition. Moreover, we readily notice that any equidistant sequence of partitions satisfies both conditions.

Next, for any $k, n \in \mathbb{N}$ we are interested in the delayed linear interpolation of a map $x:[0, T] \rightarrow \mathbb{R}^{k}$ along $\mathbb{T}_{n}$. Namely, we define $L_{n}(x):[0, T] \rightarrow \mathbb{R}^{k}$ by $L_{n}(x)(t):=x(r \wedge t)$, if $t \leq t_{1, n}$, and

$$
L_{n}(x)(t):=x\left(t_{i-1, n}\right)+\frac{t-t_{i, n}}{t_{i+1, n}-t_{i, n}}\left(x\left(t_{i, n}\right)-x\left(t_{i-1, n}\right)\right),
$$

if $t \in\left(t_{i, n}, t_{i+1, n}\right]$ for some $i \in\left\{1, \ldots, k_{n}-1\right\}$. Since $L_{n}(x)$ is piecewise continuously differentiable on $[r, T]$, it belongs to $W_{r}^{1, p}\left([0, T], \mathbb{R}^{k}\right)$ for each $p \geq 1$ if $x$ is continuous on $[0, r]$, and by construction, the process ${ }_{n} W:[0, T] \times \Omega \rightarrow \mathbb{R}^{d}$ defined via ${ }_{n} W_{t}:=L_{n}(W)(t)$ is adapted.

Let us now assume that (C.1)-(C.3) and Lemma 1.1 hold. Then the support of $P \circ X^{-1}$ is included in the closure of $\left\{x_{h} \mid h \in W_{r}^{1, p}\left([0, T], \mathbb{R}^{d}\right)\right\}$ in $C_{r}^{\alpha}\left([0, T], \mathbb{R}^{m}\right)$ for $\alpha \in[0,1 / 2)$ and $p \geq 2$ if we can prove that

$$
\lim _{n \uparrow \infty} P\left(\left\|x_{n} W-X\right\|_{\alpha, r} \geq \varepsilon\right)=0 \text { for any } \varepsilon>0 .
$$

Moreover, if for each $h \in W_{r}^{1, p}\left([0, T], \mathbb{R}^{d}\right)$ there exists a sequence $\left(P_{h, n}\right)_{n \in \mathbb{N}}$ of probability measures on $(\Omega, \mathscr{F})$ that are absolutely continuous to $P$ such that

$$
\lim _{n \uparrow \infty} P_{h, n}\left(\left\|X-x_{h}\right\|_{\alpha, r} \geq \varepsilon\right)=0 \text { for every } \varepsilon>0,
$$

then the converse inclusion holds. The sufficiency of (2.4) and (2.5) follows from a basic result on the support of probability measures, see [7][Lemma 36] for example. To verify the validity of both limits, we consider a more general setting.

Let $\underline{B}$ be an $\mathbb{R}^{m}$-valued and $B_{H}, \bar{B}$ and $\Sigma$ be $\mathbb{R}^{m \times d}$-valued non-anticipative product measurable maps on $[r, T]^{2} \times C\left([0, T], \mathbb{R}^{m}\right)$. For any $n \in \mathbb{N}$ we study the path-dependent stochastic Volterra integral equation:

$$
\begin{aligned}
{ }_{n} Y_{t}= & { }_{n} Y_{r}+\int_{r}^{t} \underline{B}\left(t, s,{ }_{n} Y\right)+B_{H}\left(t, s,{ }_{n} Y\right) \dot{h}(s)+\bar{B}\left(t, s,{ }_{n} Y\right)_{n} \dot{W}_{s} d s \\
& +\int_{r}^{t} \Sigma\left(t, s,{ }_{n} Y\right) d W_{s} \quad \text { a.s. for } t \in[r, T] .
\end{aligned}
$$

Provided that the map $[r, t) \times C\left([0, T], \mathbb{R}^{m}\right) \rightarrow \mathbb{R}^{m \times d},(s, x) \mapsto \bar{B}(t, s, x)$ is of class $\mathbb{C}^{1,2}$ for all $t \in(r, T]$, we introduce another path-dependent stochastic Volterra integral equation:

$$
\begin{aligned}
Y_{t}= & Y_{r}+\int_{r}^{t}(\underline{B}+R)(t, s, Y)+B_{H}(t, s, Y) \dot{h}(s) d s \\
& +\int_{r}^{t}(\bar{B}+\Sigma)(t, s, Y) d W_{s} \quad \text { a.s. for } t \in[r, T]
\end{aligned}
$$


with the $\mathbb{R}^{m}$-valued non-anticipative product measurable map $R$ on $[r, T]^{2} \times C\left([0, T], \mathbb{R}^{m}\right)$ given coordinatewise by

$$
R_{k}(t, s, x)=\sum_{l=1}^{d} \partial_{x} \bar{B}_{k, l}(t, s, x)((1 / 2) \bar{B}+\Sigma)(s, s, x) e_{l},
$$

if $s<t$, and $R_{k}(t, s, x):=0$, otherwise. In particular, (2.6) reduces to (2.7) in the case that $\bar{B}=0$. We seek to show that if ${ }_{n} Y$ and $Y$ are two solutions to (2.6) and (2.7), respectively, satisfying ${ }_{n} Y^{r}=Y^{r}=\hat{x}^{r}$ a.s. for all $n \in \mathbb{N}$, then

$$
\lim _{n \uparrow \infty} E\left[\left\|_{n} Y-Y\right\|_{\alpha, r}^{2}\right]=0 .
$$

Thus, by choosing $\underline{B}=b-(1 / 2) \rho, B_{H}=0, \bar{B}=\sigma$ and $\Sigma=0$, we obtain (2.4). If instead $\underline{B}=b, B_{H}=\sigma, \bar{B}=-\sigma$ and $\Sigma=\sigma$, then (2.5) is implied, as we will see. To derive the general convergence result (2.9), we introduce the following regularity conditions:

(C.5) The map $[r, t) \times C\left([0, T], \mathbb{R}^{m}\right) \rightarrow \mathbb{R}^{m \times d},(s, x) \mapsto \bar{B}(t, s, x)$ is of class $\mathbb{C}^{1,2}$ for all $t \in(r, T]$, for any $F \in\left\{\underline{B}, B_{H}, \bar{B}, \Sigma\right\}$ the map $F(\cdot, s, x)$ is absolutely continuous on $[s, T]$ and $\partial_{x} \bar{B}$ is absolutely continuous on $(s, T]$ for all $(s, x) \in[r, T) \times C\left([0, T], \mathbb{R}^{m}\right)$.

(C.6) There are $c \geq 0$ and $\kappa \in[0,1)$ such that any two maps $F \in\left\{\underline{B}, B_{H}\right\}$ and $G \in\{\bar{B}, \Sigma\}$ satisfy $|F(s, s, x)|+\left|\partial_{t} F(t, s, x)\right| \leq c\left(1+\|x\|_{\infty}^{\kappa}\right)$ and $|G(s, s, x)|+\left|\partial_{t} G(t, s, x)\right| \leq c$ for all $s, t \in[r, T)$ with $s<t$ and every $x \in C\left([0, T], \mathbb{R}^{m}\right)$.

(C.7) There exists $\lambda \geq 0$ such that $|\underline{B}(s, s, x)-\underline{B}(s, s, y)|+\left|\partial_{t} \underline{B}(t, s, x)-\partial_{t} \underline{B}(t, s, y)\right|$ $\leq \lambda\|x-y\|_{\infty}$ and for any $F \in\left\{B_{H}, \bar{B}, \Sigma\right\}$ it holds that

$$
|F(u, t, x)-F(u, s, y)|+\left|\partial_{u} F(u, t, x)-\partial_{u} F(u, s, y)\right| \leq \lambda d_{\infty}((t, x),(s, y))
$$

for each $s, t, u \in[r, T)$ with $s<t<u$ and every $x, y \in C\left([0, T], \mathbb{R}^{m}\right)$.

(C.8) There are $\bar{c}, \eta, \bar{\lambda} \geq 0$ such that $\left\|\partial_{x} \bar{B}(s, s, x)\right\|+\left\|\partial_{t} \partial_{x} \bar{B}(t, s, x)\right\| \leq \bar{c},\left|\partial_{s} \bar{B}(t, s, x)\right|$ $+\left\|\partial_{x x} \bar{B}(t, s, x)\right\| \leq \bar{c}\left(1+\|x\|_{\infty}^{\eta}\right)$ and

$$
\left\|\partial_{x} \bar{B}(u, t, x)-\partial_{x} \bar{B}(u, s, y)\right\| \leq \bar{\lambda} d_{\infty}((t, x),(s, y))
$$

for any $s, t, u \in[r, T)$ with $s<t<u$ and all $x, y \in C\left([0, T], \mathbb{R}^{m}\right)$.

(C.9) The map $\Sigma$ vanishes or there is a measurable function $\bar{b}:[r, T] \rightarrow \mathbb{R}$ such that $\int_{r}^{T} \bar{b}(s)^{2} d s<\infty$ and $\bar{B}=\bar{b} \Sigma$.

First, we question uniqueness, existence and regularity of solutions to (2.6) and (2.7). In this regard, let $\xi \in \mathscr{C}\left([0, T], \mathbb{R}^{m}\right)$ and $\left.{ }_{n} \xi\right)_{n \in \mathbb{N}}$ be a sequence in $\mathscr{C}\left([0, T], \mathbb{R}^{m}\right)$.

Lemma 2.2. Assume that (C.5)-(C.7) are satisfied, $h \in W_{r}^{1,2}\left([0, T], \mathbb{R}^{d}\right)$ and there is a sequence $\left(p_{n}\right)_{n \in \mathbb{N}_{0}}$ in $(2, \infty)$ such that $E\left[\left\|\xi^{r}\right\|_{\infty}^{p_{0}}+\left\|_{n} \xi^{r}\right\|_{\infty}^{p_{n}}\right]<\infty$ for all $n \in \mathbb{N}$.

(i) Under (C.9), pathwise uniqueness holds for (2.6) and there exists a unique strong solution ${ }_{n} Y$ with ${ }_{n} Y^{r}={ }_{n} \xi^{r}$ a.s. for any $n \in \mathbb{N}$. Further, for each $p>2$ and every $\alpha \in[0,1 / 2-1 / p)$, there is $c_{\alpha, p}>0$ such that

$$
E\left[\left\|_{n} Y\right\|_{\alpha, r}^{p}\right] \leq c_{\alpha, p}\left(1+E\left[\left\|_{n} \xi^{r}\right\|_{\infty}^{p}\right]\right) \text { for all } n \in \mathbb{N} .
$$

(ii) If (C.8) holds, then we have pathwise uniqueness for (2.7) and a unique strong solution $Y$ with $Y^{r}=\xi^{r}$ a.s. In this case, for each $p>2$ and all $\alpha \in[0,1 / 2-1 / p)$ there is $\bar{c}_{\alpha, p}>0$ with $E\left[\|Y\|_{\alpha, r}^{p}\right] \leq \bar{c}_{\alpha, p}\left(1+E\left[\left\|\xi^{r}\right\|_{\infty}^{p}\right]\right)$. 
Finally, we consider a convergence result in Hölder norms in second moment.

Theorem 2.3. Let (C.4)-(C.9) hold, $h \in W_{r}^{1,2}\left([0, T], \mathbb{R}^{d}\right)$ and $\alpha \in[0,1 / 2)$. Suppose that $\lim _{n \uparrow \infty} E\left[\left\|{ }_{n} \xi^{r}-\xi^{r}\right\|_{\infty}^{2}\right] /\left|\mathbb{T}_{n}\right|^{2 \alpha}=0$ and there is $p>2$ such that

$$
\alpha<1 / 2-1 / p \text { and } E\left[\left\|\xi^{r}\right\|_{\infty}^{p}\right]+\sup _{n \in \mathbb{N}} E\left[\left\|_{n} \xi^{r}\right\|_{\infty}^{(2 \vee \eta) p}\right]<\infty .
$$

Let ${ }_{n} Y$ and $Y$ be the unique strong solutions to (2.6) and (2.7), respectively, such that ${ }_{n} Y^{r}={ }_{n} \xi^{r}$ and $Y^{r}=\xi^{r}$ a.s. for all $n \in \mathbb{N}$, then

$$
\lim _{n \uparrow \infty} E\left[\max _{j \in\left\{0, \ldots, k_{n}\right\}}\left|{ }_{n} Y_{t_{j, n}}-Y_{t_{j, n}}\right|^{2}\right] /\left|\mathbb{T}_{n}\right|^{2 \alpha}=0 .
$$

In particular, (2.9) is satisfied. That is, $\left({ }_{n} Y\right)_{n \in \mathbb{N}}$ converges in the delayed $\alpha$-Hölder norm $\|\cdot\|_{\alpha, r}$ in second moment to $Y$.

\section{Estimates for convergence in Hölder norms in moments}

\subsection{Convergence in moments along a sequence of partitions}

We consider a sufficient condition for a sequence of processes to converge in the norm $\|\cdot\|_{\alpha, r}$ in $p$-th moment, where $\alpha \in[0,1]$ and $p \geq 1$. Its derivation relies on an explicit Kolmogorov-Chentsov estimate from [7][Proposition 12].

Namely, let $X$ be an $\mathbb{R}^{m}$-valued right-continuous process for which there are $c_{0} \geq 0$, $p \geq 1$ and $q>0$ such that $E\left[\left|X_{s}-X_{t}\right|^{p}\right] \leq c_{0}|s-t|^{1+q}$ for all $s, t \in[r, T]$. Then it follows that

$$
E\left[\sup _{s, t \in[r, T]: s \neq t} \frac{\left|X_{s}-X_{t}\right|^{p}}{|s-t|^{\alpha p}}\right] \leq k_{\alpha, p, q} c_{0}(T-r)^{1+q-\alpha p}
$$

for any $\alpha \in[0, q / p)$ with $k_{\alpha, p, q}:=2^{p+q}\left(2^{q / p-\alpha}-1\right)^{-p}$. In particular, if $q \leq p$, then $X$ itself, and not necessarily a modification, admits a.s. $\alpha$-Hölder continuous paths on $[r, T]$.

Lemma 3.1. Let $\left({ }_{n} X\right)_{n \in \mathbb{N}}$ be a sequence of $\mathbb{R}^{m}$-valued right-continuous processes for which there are $c_{0} \geq 0, p \geq 1$ and $q>0$ with $q \leq p$ such that

$$
E\left[{ }_{n} X_{s}-\left.{ }_{n} X_{t}\right|^{p}\right] \leq c_{0}|s-t|^{1+q}
$$

for all $n \in \mathbb{N}$, each $j \in\left\{0, \ldots, k_{n}-1\right\}$ and any $s, t \in\left[t_{j, n}, t_{j+1, n}\right]$. If $\left(\left\|_{n} X^{r}\right\|_{\infty}\right)_{n \in \mathbb{N}}$ and $\left(\max _{j \in\left\{1, \ldots, k_{n}\right\}}\left|{ }_{n} X_{t_{j, n}}\right| /\left|\mathbb{T}_{n}\right|^{\alpha}\right)_{n \in \mathbb{N}}$ converge in $p_{0}$-th moment to zero for some $p_{0} \in[1, p]$, then so does the sequence $\left(\left\|_{n} X\right\|_{\alpha, r}\right)_{n \in \mathbb{N}}$ for every $\alpha \in[0, q / p)$.

Proof. For given $n \in \mathbb{N}$ a case distinction yields that

$$
\begin{aligned}
\sup _{s, t \in[r, T]: s \neq t} \frac{\left|{ }_{n} X_{s}-{ }_{n} X_{t}\right|}{|s-t|^{\alpha}} \leq & 2 \max _{j \in\left\{0, \ldots, k_{n}-1\right\}} \sup _{s, t \in\left[t_{j, n}, t_{j+1, n}\right]: s \neq t} \frac{\left|{ }_{n} X_{s}-{ }_{n} X_{t}\right|}{|s-t|^{\alpha}} \\
& +\max _{i, j \in\left\{1, \ldots, k_{n}\right\}: i \neq j} \frac{\left|{ }_{n} X_{t_{i, n}}-{ }_{n} X_{t_{j, n}}\right|}{\left|t_{i, n}-t_{j, n}\right|^{\alpha}} .
\end{aligned}
$$

By virtue of the Kolmogorov-Chentsov estimate (3.1), it holds that

$$
E\left[\max _{j \in\left\{0, \ldots, k_{n}-1\right\}} \sup _{s, t \in\left[t_{j, n}, t_{j+1, n}\right]: s \neq t} \frac{\left|{ }_{n} X_{s}-{ }_{n} X_{t}\right|^{p}}{|s-t|^{\alpha p}}\right] \leq k_{\alpha, p, q} c_{0}(T-r)\left|\mathbb{T}_{n}\right|^{q-\alpha p},
$$

since $q>\alpha p$ and $\sum_{j=0}^{k_{n}-1}\left(t_{j+1, n}-t_{j, n}\right)=T-r$. Moreover, from condition (2.2) we infer that $\left|t_{i, n}-t_{j, n}\right| \geq\left|\mathbb{T}_{n}\right| / c_{\mathbb{T}}$ for all $i, j \in\left\{0, \ldots, k_{n}\right\}$ with $i \neq j$. Hence,

$$
E\left[\max _{i, j \in\left\{1, \ldots, k_{n}\right\}: i \neq j} \frac{\left|{ }_{n} X_{t_{i, n}}-{ }_{n} X_{t_{j, n}}\right|^{p_{0}}}{\left|t_{i, n}-t_{j, n}\right|^{\alpha p_{0}}}\right] \leq 2^{p_{0}-1} c_{\mathrm{T}}^{\alpha p_{0}} E\left[\max _{j \in\left\{1, \ldots, k_{n}\right\}}\left|{ }_{n} X_{t_{j, n}}\right|^{p_{0}}\right] /\left|\mathbb{T}_{n}\right|^{\alpha p_{0}} .
$$

Now the claim follows from the definition of the norm $\|\cdot\|_{\alpha, r}$ and Hölder's inequality. 


\subsection{Sequential notations and auxiliary moment estimates}

Let us introduce relevant notations related to the sequence of partitions $\left(\mathbb{T}_{n}\right)_{n \in \mathbb{N}}$. For fixed $n \in \mathbb{N}$ and $t \in[r, T)$, we choose $i \in\left\{0, \ldots, k_{n}-1\right\}$ such that $t \in\left[t_{i, n}, t_{i+1, n}\right)$ and set

$$
\underline{t}_{n}:=t_{(i-1) \vee 0, n}, \quad t_{n}:=t_{i, n} \quad \text { and } \quad \bar{t}_{n}:=t_{i+1, n} .
$$

Verbalized, $\underline{t}_{n}$ is the predecessor of $t_{n}$ relative to $\mathbb{T}_{n}$, provided $i \neq 0$, and $\bar{t}_{n}$ is the successor of $t_{n}$. For the sake of completeness, let $\underline{T}_{n}:=t_{k_{n-1}, n}, T_{n}:=T$ and $\bar{T}_{n}:=T$. Further, for $i \in\left\{0, \ldots, k_{n}\right\}$ we set

$$
\Delta t_{i, n}:=t_{i, n}-t_{(i-1) \vee 0, n} \quad \text { and } \quad \Delta W_{t_{i, n}}:=W_{t_{i, n}}-W_{t_{(i-1) \vee 0, n}} .
$$

For $p \geq 1$ we recall an interpolation error estimate in supremum norm in $p$-th moment and an explicit integral moment estimate for the sequence $\left({ }_{n} W\right)_{n \in \mathbb{N}}$ of adapted linear interpolations of $W$ from [7][Lemmas 19 and 17].

(i) Let $\left({ }_{n} X\right)_{n \in \mathbb{N}}$ be a sequence of $\mathbb{R}^{m}$-valued right-continuous processes for which there are $c_{0} \geq 0$ and $q>0$ such that $E\left[{ }_{n} X_{s}-\left.{ }_{n} X_{t}\right|^{p}\right] \leq c_{0}|s-t|^{1+q}$ for all $n \in \mathbb{N}$, each $j \in\left\{0, \ldots, k_{n}-1\right\}$ and every $s, t \in\left[t_{j, n}, t_{j+1, n}\right]$. Then there is $c_{p, q}>0$ such that

$$
E\left[\left\|L_{n}\left({ }_{n} X\right)-{ }_{n} X\right\|_{\infty}^{p}\right] \leq c_{p, q} c_{0}\left|\mathbb{T}_{n}\right|^{q}
$$

for all $n \in \mathbb{N}$. To be precise, $c_{p, q}=2^{p-1}\left(1+k_{0, p, q}\right)(T-r)$.

(ii) Let $q \geq 1$ and $Z$ be an $\mathbb{R}^{d}$-valued random vector such that $Z \sim \mathcal{N}\left(0, \mathbb{I}_{d}\right)$. Then the constant $\hat{w}_{p, q}:=E\left[|Z|^{p q}\right] c_{\mathrm{T}}^{p q}$ satisfies

$$
E\left[\left(\left.\left.\int_{s}^{t}\right|_{n} \dot{W}_{u}\right|^{q} d u\right)^{p}\right] \leq \hat{w}_{p, q}\left|\mathbb{T}_{n}\right|^{-\frac{p q}{2}}(t-s)^{p}
$$

for all $n \in \mathbb{N}$ and every $s, t \in[r, T]$ with $s \leq t$.

Next, we let $p \geq 2$ and state a Burkholder-Davis-Gundy inequality for stochastic integrals with respect to $W$ from [10][Theorem 7.2]. Based on this bound, one can deduce an estimate for integrals relative to ${ }_{n} W$ that is independent of $n \in \mathbb{N}$ and which is given in [7][Proposition 16].

(iii) For each $\mathbb{R}^{m \times d}$-valued progressively measurable process $X$ for which $\int_{r}^{T} E\left[\left|X_{u}\right|^{p}\right] d u$ is finite,

$$
E\left[\sup _{v \in[s, t]}\left|\int_{s}^{v} X_{u} d W_{u}\right|^{p}\right] \leq w_{p}(t-s)^{\frac{p}{2}-1} \int_{s}^{t} E\left[\left|X_{u}\right|^{p}\right] d u
$$

for all $s, t \in[r, T]$ with $s \leq t$ and $w_{p}:=\left(\left(p^{3} / 2\right) /(p-1)\right)^{p / 2}$.

(iv) Any $\mathbb{R}^{m \times d}$-valued progressively measurable process $X$ satisfies

$$
E\left[\max _{v \in[s, t]}\left|\int_{s}^{v} X_{\underline{u}_{n}} d_{n} W_{u}\right|^{p}\right] \leq \hat{w}_{p}(t-s)^{\frac{p}{2}} \max _{j \in\left\{0, \ldots, k_{n}\right\}: t_{j, n} \in\left[\underline{s}_{n}, \underline{t}_{n}\right]} E\left[\left|X_{t_{j, n}}\right|^{p}\right]
$$

for each $n \in \mathbb{N}$ and all $s, t \in[r, T]$ with $s \leq t$, where $\hat{w}_{p}:=3^{p} w_{p} c_{\mathbb{T}}^{p / 2}$.

\subsection{Moment estimates for Volterra integrals}

The first integral bound that we consider follows from the auxiliary estimate (3.3). 
Lemma 3.2. Let $p>1$ and assume that ${ }_{n} X:[r, T]^{2} \times \Omega \rightarrow \mathbb{R}_{+},(t, s, \omega) \mapsto X_{t, s}(\omega)$ is a product measurable function for each $n \in \mathbb{N}$. If there are $p_{0}>p, c_{p_{0}}>0$ and $q \geq p_{0} / 2$ such that

$$
E\left[\max _{j \in\left\{0, \ldots, k_{n}\right\}} \int_{r}^{t_{j, n}}{ }_{n} X_{t_{j, n}, s}^{p_{0}} d s\right] \leq c_{p_{0}}\left|\mathbb{T}_{n}\right|^{q} \quad \text { for all } n \in \mathbb{N} .
$$

Then there is $c_{p}>0$ such that

$$
E\left[\max _{j \in\left\{0, \ldots, k_{n}\right\}}\left(\left.\int_{r}^{t_{j, n}}{ }_{n} X_{t_{j, n}, s}\right|_{n} \dot{W}_{s} \mid d s\right)^{p}\right] \leq c_{p}\left|\mathbb{T}_{n}\right|^{p\left(\frac{q}{p_{0}}-\frac{1}{2}\right)} \quad \text { for any } n \in \mathbb{N} .
$$

Proof. Let $q_{1}$ and $q_{2}$ denote the dual exponents of $p$ and $p_{0} / p$, respectively, and set $p_{1}:=\max \left\{(p-1) q_{2}, 1\right\}$. Then three applications of Hölder's inequality yield that

$$
\begin{aligned}
E\left[\max _{j \in\left\{0, \ldots, k_{n}\right\}}\left(\left.\int_{r}^{t_{j, n}}{ }_{n} X_{t_{j, n}, s}\right|_{n} \dot{W}_{s} \mid d s\right)^{p}\right] \\
\leq E\left[\max _{j \in\left\{0, \ldots, k_{n}\right\}}\left(\int_{r}^{t_{j, n}}{ }_{n} X_{t_{j, n}, s}^{p} d s\right)^{\frac{p_{0}}{p}}\right]^{\frac{p}{p_{0}}} c_{p, 1}\left|\mathbb{T}_{n}\right|^{-\frac{p}{2}}
\end{aligned}
$$

with $c_{p, 1}:=\hat{w}_{p_{1}, q_{1}}^{(p-1) / p_{1}}(T-r)^{p-1}$, where $\hat{w}_{p_{1}, q_{1}}$ is the constant introduced at (3.3). For this reason, the constant $c_{p}:=(T-r)^{1-p / p_{0}} c_{p_{0}}^{p / p_{0}} c_{p, 1}$ satisfies the desired estimate.

Remark 3.3. For any $n \in \mathbb{N}$ let ${ }_{n} X$ be independent of the first time variable, that is, there is an $\mathbb{R}_{+}$-valued measurable process ${ }_{n} Y$ with ${ }_{n} X_{t, s}={ }_{n} Y_{s}$ for all $s, t \in[r, T]$. Then for condition (3.6) to hold, it suffices that there is $\bar{c}_{p_{0}}>0$ so that $E\left[{ }_{n} Y_{s}^{p_{0}}\right] \leq \bar{c}_{p_{0}}\left|\mathbb{T}_{n}\right|^{q}$ for each $n \in \mathbb{N}$ and every $s \in[r, T)$.

For the second and various other estimates in the following section, let us use for each $n \in \mathbb{N}$ the function $\gamma_{n}:[r, T] \rightarrow\left[0, c_{\mathbb{T}}\right]$ defined by

$$
\gamma_{n}(s):=\frac{\Delta s_{n}}{\Delta \bar{s}_{n}} .
$$

Put differently, $\gamma_{n}=\Delta t_{i, n} / \Delta t_{i+1, n}$ on $\left[t_{i, n}, t_{i+1, n}\right)$ for all $i \in\left\{0, \ldots, k_{n}-1\right\}$ and $\gamma_{n}(T)=1$. Lemma 3.4. Assume that $F:[r, T]^{2} \times C\left([0, T], \mathbb{R}^{m}\right) \rightarrow \mathbb{R}^{m}$ is a non-anticipative product measurable map for which there are $c_{0}, \lambda_{0} \geq 0$ such that

$$
|F(t, s, x)| \leq c_{0}\left(1+\|x\|_{\infty}\right) \quad \text { and } \quad|F(u, t, x)-F(u, s, x)| \leq \lambda_{0} d_{\infty}((t, x),(s, x))
$$

for all $s, t, u \in[r, T]$ with $s<t<u$ and each $x \in C\left([0, T], \mathbb{R}^{m}\right)$. Further, let $\left({ }_{n} Y\right)_{n \in \mathbb{N}}$ be a sequence in $\mathscr{C}\left([0, T], \mathbb{R}^{m}\right)$ which there are $p \geq 1$ and $c_{p, 0} \geq 0$ such that

$$
E\left[\left\|_{n} Y\right\|_{\infty}^{p}\right]+E\left[\left\|_{n} Y^{s}-{ }_{n} Y^{t}\right\|_{\infty}^{p}\right] /|s-t|^{\frac{p}{2}} \leq c_{p, 0}\left(1+E\left[\left\|_{n} Y^{r}\right\|_{\infty}^{p}\right]\right)
$$

for each $n \in \mathbb{N}$ and any $s, t \in[r, T]$ with $s<t$. Then there is $c_{p}>0$ such that

$$
E\left[\max _{j \in\left\{0, \ldots, k_{n}\right\}}\left|\int_{r}^{t_{j, n}} F\left(t_{j, n}, \underline{s}_{n},{ }_{n} Y\right)\left(\gamma_{n}(s)-1\right) d s\right|^{p}\right] \leq c_{p}\left|\mathbb{T}_{n}\right|^{\frac{p}{2}}\left(1+E\left[\left\|_{n} Y^{r}\right\|_{\infty}^{p}\right]\right)
$$

for every $n \in \mathbb{N}$.

Proof. Let $E\left[\left\|_{n} Y^{r}\right\|_{\infty}^{p}\right]<\infty$, as otherwise the claimed estimate is infinite. Clearly, a decomposition of the integral shows that

$$
\int_{r}^{t_{j, n}} F\left(t_{j, n}, \underline{s}_{n},{ }_{n} Y\right) \gamma_{n}(s) d s=\int_{r}^{t_{j-1, n}} F\left(t_{j, n}, s_{n},{ }_{n} Y\right) d s
$$


for all $j \in\left\{1, \ldots, k_{n}\right\}$. Hence, a first estimation gives

$$
E\left[\max _{j \in\left\{1, \ldots, k_{n}\right\}}\left|\int_{r}^{t_{j-1, n}} F\left(t_{j, n}, s_{n},{ }_{n} Y\right)-F\left(t_{j, n}, \underline{s}_{n},{ }_{n} Y\right) d s\right|^{p}\right] \leq c_{p, 1}\left|\mathbb{T}_{n}\right|^{\frac{p}{2}}\left(1+E\left[\left\|_{n} Y^{r}\right\|_{\infty}^{p}\right]\right)
$$

for $c_{p, 1}:=2^{p-1}(T-r)^{p} \lambda_{0}^{p}\left(1+c_{p, 0}\right)$ and a second yields that

$$
E\left[\max _{j \in\left\{1, \ldots, k_{n}\right\}}\left|\int_{t_{j-1, n}}^{t_{j, n}} F\left(t_{j, n}, \underline{s}_{n}, n Y\right) d s\right|^{p}\right] \leq c_{p, 2}\left|\mathbb{T}_{n}\right|^{p}\left(1+E\left[\left\|_{n} Y^{r}\right\|_{\infty}^{p}\right]\right)
$$

with $c_{p, 2}:=2^{p-1} c_{0}^{p}\left(1+c_{p, 0}\right)$. Thus, the constant $c_{p}:=2^{p-1}\left(c_{p, 1}+(T-r)^{p / 2} c_{p, 2}\right)$ satisfies the asserted estimate.

The third estimate deals with Volterra integrals driven by ${ }_{n} W$ and $W$, where $n \in \mathbb{N}$.

Proposition 3.5. Let $F:[r, T]^{2} \times C\left([0, T], \mathbb{R}^{m}\right) \rightarrow \mathbb{R}^{m \times d}$ be non-anticipative, product measurable and such that $F(\cdot, s, x)$ is absolutely continuous on $[s, T]$ for all $s \in[r, T]$ and each $x \in C\left([0, T], \mathbb{R}^{m}\right)$. Suppose that there are $c_{0}, \lambda_{0} \geq 0$ such that

$$
\begin{aligned}
|F(s, s, x)|+\left|\partial_{t} F(t, s, x)\right| & \leq c_{0}\left(1+\|x\|_{\infty}\right) \quad \text { and } \\
|F(u, t, x)-F(u, s, x)|+\left|\partial_{u} F(u, t, x)-\partial_{u} F(u, s, x)\right| & \leq \lambda_{0} d_{\infty}((t, x),(s, x))
\end{aligned}
$$

for any $s, t, u \in[r, T)$ with $s<t<u$ and every $x \in C\left([0, T], \mathbb{R}^{m}\right)$. Moreover, let $\left({ }_{n} Y\right)_{n \in \mathbb{N}}$ be a sequence in $\mathscr{C}\left([0, T], \mathbb{R}^{m}\right)$ for which there are $p \geq 2$ and $c_{p, 0} \geq 0$ such that

$$
E\left[\left\|_{n} Y\right\|_{\infty}^{p}\right]+E\left[\left\|_{n} Y^{s}-{ }_{n} Y^{t}\right\|_{\infty}^{p}\right] /|s-t|^{\frac{p}{2}} \leq c_{p, 0}\left(1+E\left[\left\|_{n} Y^{r}\right\|_{\infty}^{p}\right]\right)
$$

for all $n \in \mathbb{N}$ and each $s, t \in[r, T]$ with $s<t$. Then there is $c_{p}>0$ such that

$$
E\left[\max _{j \in\left\{0, \ldots, k_{n}\right\}}\left|\int_{r}^{t_{j, n}} F\left(t_{j, n}, \underline{s}_{n},{ }_{n} Y\right) d\left({ }_{n} W_{s}-W_{s}\right)\right|^{p}\right] \leq c_{p}\left|\mathbb{T}_{n}\right|^{\frac{p}{2}-1}\left(1+E\left[\left\|_{n} Y^{r}\right\|_{\infty}^{p}\right]\right)
$$

for every $n \in \mathbb{N}$.

Proof. We suppose that $E\left[\left\|_{n} Y^{r}\right\|_{\infty}^{p}\right]$ is finite and decompose the integral to get that

$$
\int_{r}^{t_{j, n}} F\left(t_{j, n}, \underline{s}_{n},{ }_{n} Y\right) d_{n} W_{s}=\int_{r}^{t_{j-1, n}} F\left(t_{j, n}, s_{n},{ }_{n} Y\right) d W_{s} \quad \text { a.s. }
$$

for each $j \in\left\{1, \ldots, k_{n}\right\}$. Hence, we may apply Fubini's theorem for stochastic integrals from [15] to obtain that

$$
\begin{aligned}
\int_{r}^{t_{j-1, n}} F\left(t_{j, n}, s_{n},{ }_{n} Y\right) & -F\left(t_{j, n}, \underline{s}_{n},{ }_{n} Y\right) d W_{s}=\int_{r}^{t_{j-1, n}} F\left(s, s_{n},{ }_{n} Y\right)-F\left(s, \underline{s}_{n},{ }_{n} Y\right) d W_{s} \\
& +\int_{r}^{t_{j, n}} \int_{r}^{t \wedge t_{j-1, n}} \partial_{t} F\left(t, s_{n},{ }_{n} Y\right)-\partial_{t} F\left(t, \underline{s}_{n},{ }_{n} Y\right) d W_{s} d t \quad \text { a.s. }
\end{aligned}
$$

for all $j \in\left\{1, \ldots, k_{n}\right\}$. Regarding the first expression, we estimate that

$$
E\left[\max _{j \in\left\{1, \ldots, k_{n}\right\}}\left|\int_{r}^{t_{j-1, n}} F\left(s, s_{n},{ }_{n} Y\right)-F\left(s, \underline{s}_{n},{ }_{n} Y\right) d W_{s}\right|^{p}\right] \leq c_{p, 1}\left|\mathbb{T}_{n}\right|^{\frac{p}{2}}\left(1+E\left[\|\|_{n} Y^{r} \|_{\infty}^{p}\right]\right)
$$

for $c_{p, 1}:=2^{p-1} w_{p}(T-r)^{p / 2} \lambda_{0}^{p}\left(1+c_{p, 0}\right)$, where $w_{p}$ is the constant satisfying (3.4). For the second expression we first calculate that

$$
E\left[\max _{j \in\left\{1, \ldots, k_{n}\right\}}\left|\int_{r}^{t_{j-1, n}} \int_{r}^{t} \partial_{t} F\left(t, s_{n},,_{n} Y\right)-\partial_{t} F\left(t, \underline{s}_{n},{ }_{n} Y\right) d W_{s} d t\right|^{p}\right]
$$


Support characterization for stochastic Volterra integral equations

$$
\leq c_{p, 2}\left|\mathbb{T}_{n}\right|^{\frac{p}{2}}\left(1+E\left[\left\|_{n} Y^{r}\right\|_{\infty}^{p}\right]\right)
$$

with $c_{p, 2}:=2^{p}(p+2)^{-1} w_{p}(T-r)^{3 p / 2} \lambda_{0}^{p}\left(1+c_{p, 0}\right)$. And secondly,

$$
\begin{aligned}
& E\left[\max _{j \in\left\{1, \ldots, k_{n}\right\}}\left|\int_{t_{j-1, n}}^{t_{j, n}} \int_{r}^{t_{j-1, n}} \partial_{t} F\left(t, s_{n},{ }_{n} Y\right)-\partial_{t} F\left(t, \underline{s}_{n},{ }_{n} Y\right) d W_{s} d t\right|^{p}\right] \\
& \leq \sum_{j=1}^{k_{n}}\left(t_{j, n}-t_{j-1, n}\right)^{p-1} \int_{t_{j-1, n}}^{t_{j, n}} E\left[\left|\int_{r}^{t_{j-1, n}} \partial_{t} F\left(t, s_{n},{ }_{n} Y\right)-\partial_{t} F\left(t, \underline{s}_{n},{ }_{n} Y\right) d W_{s}\right|^{p}\right] d t \\
& \leq c_{p, 3}\left|\mathbb{T}_{n}\right|^{p-1}\left(1+E\left[\left\|_{n} Y^{r}\right\|_{\infty}^{p}\right]\right),
\end{aligned}
$$

where $c_{p, 3}:=2^{p-1} w_{p}(T-r)^{p / 2+1} \lambda_{0}^{p}\left(1+c_{p, 0}\right)$. Next, for the remaining term Fubini's theorem for stochastic integrals yields that

$$
\begin{aligned}
\int_{t_{j-1, n}}^{t_{j, n}} F\left(t_{j, n}, \underline{s}_{n},{ }_{n} Y\right) d W_{s}= & \int_{t_{j-1, n}}^{t_{j, n}} F\left(s, \underline{s}_{n}, n Y\right) d W_{s} \\
& +\int_{t_{j-1, n}}^{t_{j, n}} \int_{t_{j-1, n}}^{t} \partial_{t} F\left(t, \underline{s}_{n},{ }_{n} Y\right) d W_{s} d t \quad \text { a.s. }
\end{aligned}
$$

for any $j \in\left\{1, \ldots, k_{n}\right\}$. For the first term we have

$$
\begin{aligned}
E\left[\max _{j \in\left\{1, \ldots, k_{n}\right\}}\left|\int_{t_{j-1, n}}^{t_{j, n}} F\left(s, \underline{s}_{n},{ }_{n} Y\right) d W_{s}\right|^{p}\right] & \leq \sum_{j=1}^{k_{n}} E\left[\left|\int_{t_{j-1, n}}^{t_{j, n}} F\left(s, \underline{s}_{n},{ }_{n} Y\right) d W_{s}\right|^{p}\right] \\
& \leq c_{p, 4}\left|\mathbb{T}_{n}\right|^{\frac{p}{2}-1}\left(1+E\left[\left\|_{n} Y^{r}\right\|_{\infty}^{p}\right]\right)
\end{aligned}
$$

with $c_{p, 4}:=2^{p-1} w_{p}(T-r) c_{0}^{p}\left(1+c_{p, 0}\right)$. For the second integral in the decomposition (3.8) it holds that

$$
\begin{aligned}
E\left[\max _{j \in\left\{1, \ldots, k_{n}\right\}} \mid\right. & \left.\left.\int_{t_{j-1, n}}^{t_{j, n}} \int_{t_{j-1, n}}^{t} \partial_{t} F\left(t, \underline{s}_{n},{ }_{n} Y\right) d W_{s} d t\right|^{p}\right] \\
& \leq \sum_{j=1}^{k_{n}}\left(t_{j, n}-t_{j-1, n}\right)^{p-1} \int_{t_{j-1, n}}^{t_{j, n}} E\left[\left|\int_{t_{j-1, n}}^{t} \partial_{t} F\left(t, \underline{s}_{n},{ }_{n} Y\right) d W_{s}\right|^{p}\right] d t \\
& \leq c_{p, 5}\left|\mathbb{T}_{n}\right|^{\frac{3}{2} p-1}\left(1+E\left[\left\|_{n} Y^{r}\right\|_{\infty}^{p}\right]\right)
\end{aligned}
$$

for $c_{p, 5}:=2^{p}(p+2)^{-1} w_{p}(T-r) c_{0}^{p}\left(1+c_{p, 0}\right)$. Hence, the asserted estimate follows readily by setting $c_{p}:=5^{p-1}\left((T-r)\left(c_{p, 1}+c_{p, 2}\right)+(T-r)^{p / 2} c_{p, 3}+c_{p, 4}+(T-r)^{p} c_{p, 5}\right)$.

\section{Estimates and decompositions for the convergence result}

\subsection{Decomposition into remainder terms}

We first give a moment estimate for solutions to (2.6) that does not depend on $n \in \mathbb{N}$. Proposition 4.1. Let (C.5) and (C.6) hold, $h \in W_{r}^{1,2}\left([0, T], \mathbb{R}^{d}\right)$ and $\lambda \geq 0$ be so that

$$
|\bar{B}(u, t, x)-\bar{B}(u, s, x)|+\left|\partial_{u} \bar{B}(u, t, x)-\partial_{u} \bar{B}(u, s, x)\right| \leq \lambda d_{\infty}((t, x),(s, x))
$$

for any $s, t, u \in[r, T)$ with $s<t<u$ and every $x \in C\left([0, T], \mathbb{R}^{m}\right)$. Then for each $p \geq 2$ there is $c_{p}>0$ such that any $n \in \mathbb{N}$ and each solution ${ }_{n} Y$ to (2.6) satisfy

$$
E\left[\left\|_{n} Y\right\|_{\infty}^{p}\right]+E\left[\left\|_{n} Y^{s}-{ }_{n} Y^{t}\right\|_{\infty}^{p}\right] /|s-t|^{\frac{p}{2}} \leq c_{p}\left(1+E\left[\left\|_{n} Y^{r}\right\|_{\infty}^{p}\right]\right)
$$

for all $s, t \in[r, T]$ with $s<t$. 
Proof. We let $E\left[\left\|_{n} Y^{r}\right\|_{\infty}^{p}\right]<\infty$ and may certainly assume in (C.6) that $\kappa>0$. For given $l \in \mathbb{N}$ the stopping time $\tau_{l, n}:=\inf \left\{t \in[0, T]||_{n} Y_{t} \mid \geq l\right\} \vee r$ satisfies $\left\|_{n} Y^{\tau_{l, n}}\right\|_{\infty} \leq\left\|_{n} Y^{r}\right\|_{\infty} \vee l$ and we readily estimate that

$$
\begin{aligned}
E\left[\left\|_{n} Y^{s \wedge \tau_{l, n}}-{ }_{n} Y^{t \wedge \tau_{l, n}}\right\|_{\infty}^{p}\right]^{\frac{1}{p}} \leq & \left(\bar{c}_{p}(t-s)^{p / 2-1} \int_{s}^{t} 1+E\left[\left\|_{n} Y^{u \wedge \tau_{l, n}}\right\|_{\infty}^{\kappa p}\right] d u\right)^{\frac{1}{p}} \\
& +E\left[\sup _{v \in[s, t]}\left|\int_{s}^{v \wedge \tau_{l, n}} \bar{B}\left(u, u,{ }_{n} Y\right) d_{n} W_{u}\right|^{p}\right]^{\frac{1}{p}} \\
& +E\left[\left(\int_{s}^{t \wedge \tau_{l, n}}\left|\int_{r}^{v} \partial_{v} \bar{B}\left(v, u,{ }_{n} Y\right) d_{n} W_{u}\right| d v\right)^{p}\right]^{\frac{1}{p}}
\end{aligned}
$$

for any fixed $s, t \in[r, T]$ with $s<t$ and $\bar{c}_{p}:=6^{p-1}(1+T-r)^{p}\left((T-r)^{p / 2}+\|h\|_{1,2, r}^{p}+w_{p}\right) c^{p}$. We recall the constant $\hat{w}_{p / \kappa, 1}$ such that (3.3) holds when $p$ and $q$ are replaced by $p / \kappa$ and 1 , respectively. Then

$$
\begin{gathered}
E\left[\left(\int_{\underline{u}_{n}}^{u \wedge \tau_{l, n}}\left|\bar{B}\left(v, v,{ }_{n} Y\right)_{n} \dot{W}_{v}\right| d v\right)^{\frac{p}{\kappa}}\right]^{\kappa} \leq c_{p, 1}\left(u-\underline{u}_{n}\right)^{\frac{p}{2}} \text { and } \\
E\left[\left(\int_{\underline{u}_{n}}^{u \wedge \tau_{l, n}} \int_{r}^{v}\left|\partial_{v} \bar{B}\left(v, u^{\prime}{ }_{n} Y\right)_{n} \dot{W}_{u^{\prime}}\right| d u^{\prime} d v\right)^{\frac{p}{\kappa}}\right]^{\kappa} \leq(T-r)^{p} c_{p, 1}\left(u-\underline{u}_{n}\right)^{\frac{p}{2}}
\end{gathered}
$$

for any given $u \in[s, T]$ with the constant $c_{p, 1}:=2^{p / 2} \hat{w}_{p / \kappa, 1}^{\kappa} c^{p}$. We let $\bar{c}_{p / \kappa}$ be defined just as $\bar{c}_{p}$ above with $p$ replaced by $p / \kappa$ to get that

$$
E\left[\left\|_{n} Y^{u \wedge \tau_{l, n}}-{ }_{n} Y^{u_{n} \wedge \tau_{l, n}}\right\|_{\infty}^{\frac{p}{k}}\right]^{\kappa} \leq c_{p, 2}\left(u-\underline{u}_{n}\right)^{\frac{p}{2}}\left(1+E\left[\|\|_{n} Y^{u \wedge \tau_{l, n}} \|_{\infty}^{p}\right]\right)^{\kappa}
$$

for $c_{p, 2}:=2^{p-1}\left(\bar{c}_{p / \kappa}^{\kappa}+(1+T-r)^{p} c_{p, 1}\right)$, due to the validity of (4.2). Hence, an application of Hölder's inequality yields that

$$
\begin{gathered}
E\left[\left(\int_{s}^{t \wedge \tau_{l, n}} \mid\left(\bar{B}\left(u, u,{ }_{n} Y\right)-\bar{B}\left(\underline{u}_{n}, \underline{u}_{n},{ }_{n} Y\right)_{n} \dot{W}_{u} \mid d u\right)^{p}\right]\right. \\
\leq c_{p, 3}(t-s)^{\frac{p}{2}-1} \int_{s}^{t}\left(1+E\left[\left\|_{n} Y^{u \wedge \tau_{l, n}}\right\|_{\infty}^{p}\right]\right)^{\kappa} d u \text { and } \\
E\left[\left(\int_{s}^{t \wedge \tau_{l, n}} \int_{r}^{v}\left|\left(\partial_{v} \bar{B}\left(v, u,{ }_{n} Y\right)-\partial_{v} \bar{B}\left(v, \underline{u}_{n},{ }_{n} Y\right)\right)_{n} \dot{W}_{u}\right| d u d v\right)^{p}\right] \\
\leq(T-r)^{p} c_{p, 3}(t-s)^{\frac{p}{2}-1} \int_{s}^{t}\left(1+E\left[\left\|_{n} Y^{u \wedge \tau_{l, n}}\right\|_{\infty}^{p}\right]\right)^{\kappa} d u,
\end{gathered}
$$

where $c_{p, 3}:=2^{p / 2} 3^{p-1} \hat{w}_{(p / 2) /(1-\kappa), 2}^{1-\kappa}(T-r)^{p / 2}\left(\lambda^{p}\left(1+c_{p, 2}\right)+c^{p}(T-r)^{p / 2}\right)$. Moreover, the constant $\hat{w}_{p}$ appearing in (3.5) satisfies

$$
\begin{gathered}
E\left[\max _{v \in[s, t]}\left|\int_{s}^{v \wedge \tau_{l, n}} \bar{B}\left(\underline{u}_{n}, \underline{u}_{n},{ }_{n} Y\right) d_{n} W_{u}\right|^{p}\right] \leq \hat{w}_{p} c^{p}(t-s)^{\frac{p}{2}} \text { and } \\
E\left[\left(\int_{s}^{t \wedge \tau_{l, n}}\left|\int_{r}^{v} \partial_{v} \bar{B}\left(v, \underline{u}_{n},{ }_{n} Y\right) d_{n} W_{u}\right| d v\right)^{p}\right] \leq(T-r)^{p} \hat{w}_{p} c^{p}(t-s)^{\frac{p}{2}} .
\end{gathered}
$$

Thus, with the constant $c_{p, 4}:=3^{p-1}\left(2 \bar{c}_{p}+(1+T-r)^{p}\left(c_{p, 3}+\hat{w}_{p} c^{p}\right)\right)$ we can now infer from (4.2) that

$$
E\left[\left\|_{n} Y^{s \wedge \tau_{l, n}}-{ }_{n} Y^{t \wedge \tau_{l, n}}\right\|_{\infty}^{p}\right] \leq c_{p, 4}(t-s)^{\frac{p}{2}-1} \int_{s}^{t} 1+E\left[\left\|_{n} Y^{u \wedge \tau_{l, n}}\right\|_{\infty}^{p}\right] d u .
$$


Support characterization for stochastic Volterra integral equations

Hence, Gronwall's inequality and Fatou's lemma imply that

$$
E\left[\left\|_{n} Y^{t}\right\|_{\infty}^{p}\right] \leq \liminf _{l \uparrow \infty} E\left[\left\|_{n} Y^{t \wedge \tau_{l, n}}\right\|_{\infty}^{p}\right] \leq c_{p, 5}\left(1+E\left[\left\|_{n} Y^{r}\right\|_{\infty}^{p}\right]\right),
$$

where $c_{p, 5}:=2^{p-1}\left(1+(T-r)^{p / 2} c_{p, 4}\right) \exp \left(2^{p-1}(T-r)^{p / 2} c_{p, 4}\right)$. For this reason, we set $c_{p}:=\left(1+c_{p, 4}\right)\left(1+c_{p, 5}\right)$ and apply Fatou's lemma to (4.3), which gives the result.

Corollary 4.2. Assume (C.5), (C.6) and (C.8) and let $h \in W_{r}^{1,2}\left([0, T], \mathbb{R}^{d}\right)$. Then for every $p \geq 2$ there is $c_{p}>0$ such that each solution $Y$ to (2.7) satisfies

$$
E\left[\|Y\|_{\infty}^{p}\right]+E\left[\left\|Y^{s}-Y^{t}\right\|_{\infty}^{p}\right] /|s-t|^{\frac{p}{2}} \leq c_{p}\left(1+E\left[\left\|Y^{r}\right\|_{\infty}^{p}\right]\right)
$$

for every $s, t \in[r, T]$ with $s<t$.

Proof. As the map $R$ given by (2.8) is bounded, the assertion is a direct consequence of Proposition 4.1 by replacing $\underline{B}$ by $\underline{B}+R, \bar{B}$ by 0 and $\Sigma$ by $\bar{B}+\Sigma$.

For $n \in \mathbb{N}$ let us recall the linear operator $L_{n}$ and the function $\gamma_{n}$ given at (2.3) and (3.7), respectively, and deduce the main decomposition to establish the limit (2.10).

Proposition 4.3. Let (C.5)-(C.8) hold and $h \in W_{r}^{1,2}\left([0, T], \mathbb{R}^{d}\right)$. Then for each $p \geq 2$ there is $c_{p}>0$ such that each $n \in \mathbb{N}$ and any two solutions ${ }_{n} Y$ and $Y$ of (2.6) and (2.7), respectively, satisfy

$$
\begin{aligned}
& E\left[\max _{j \in\left\{0, \ldots, k_{n}\right\}}\left|Y_{n} Y_{t_{j, n}}-Y_{t_{j, n}}\right|^{p}\right] / c_{p} \leq\left|\mathbb{T}_{n}\right|^{\frac{p}{2}}\left(1+E\left[\left\|{ }_{n} Y^{r}\right\|_{\infty}^{p}+\left\|Y^{r}\right\|_{\infty}^{p}\right]\right) \\
& +E\left[\left\|_{n} Y^{r}-Y^{r}\right\|_{\infty}^{p}+\left\|L_{n}\left({ }_{n} Y\right)-{ }_{n} Y\right\|_{\infty}^{p}+\left\|L_{n}(Y)-Y\right\|_{\infty}^{p}\right] \\
& +E\left[\max _{j \in\left\{0, \ldots, k_{n}\right\}}\left|\int_{r}^{t_{j, n}} R\left(t_{j, n}, \underline{s}_{n},{ }_{n} Y\right)\left(\gamma_{n}(s)-1\right) d s\right|^{p}\right] \\
& +E\left[\max _{j \in\left\{0, \ldots, k_{n}\right\}}\left|\int_{r}^{t_{j, n}} \bar{B}\left(t_{j, n}, \underline{s}_{n},{ }_{n} Y\right) d\left({ }_{n} W_{s}-W_{s}\right)\right|^{p}\right] \\
& +E\left[\max _{j \in\left\{0, \ldots, k_{n}\right\}}\left|\int_{r}^{t_{j, n}}\left(\bar{B}\left(t_{j, n}, s,{ }_{n} Y\right)-\bar{B}\left(t_{j, n}, \underline{s}_{n},{ }_{n} Y\right)\right)_{n} \dot{W}_{s}-R\left(t_{j, n}, \underline{s}_{n},{ }_{n} Y\right) \gamma_{n}(s) d s\right|^{p}\right] .
\end{aligned}
$$

Proof. We suppose that $E\left[\left\|_{n} Y^{r}\right\|_{\infty}^{p}\right]$ and $E\left[\left\|Y^{r}\right\|_{\infty}^{p}\right]$ are finite and will derive the estimate by applying Gronwall's inequality to the increasing function $\varphi_{n}:[r, T] \rightarrow \mathbb{R}_{+}$given by

$$
\varphi_{n}(t):=E\left[\max _{j \in\left\{0, \ldots, k_{n}\right\}: t_{j, n} \leq t}\left|Y_{t_{j, n}}-Y_{t_{j, n}}\right|^{p}\right] .
$$

To this end, let us write the difference of ${ }_{n} Y$ and $Y$ as follows:

$$
\begin{aligned}
{ }_{n} Y_{t} & -Y_{t}={ }_{n} Y_{r}-Y_{r}+\int_{r}^{t} \underline{B}\left(s, s,{ }_{n} Y\right)-\underline{B}(s, s, Y) d s \\
& +\int_{r}^{t} B_{H}\left(s, s,{ }_{n} Y\right)-B_{H}(s, s, Y) d h(s)+{ }_{n} \Delta_{t} \\
& +\int_{r}^{t} \int_{r}^{v} \partial_{v} \underline{B}\left(v, u,{ }_{n} Y\right)-\partial_{v} \underline{B}(v, u, Y) d u d v \\
& +\int_{r}^{t} \int_{r}^{v} \partial_{v} B_{H}\left(v, u,{ }_{n} Y\right)-\partial_{v} B_{H}(v, u, Y) d h(u) d v \\
& +\int_{r}^{t} \Sigma\left(s, s,{ }_{n} Y\right)-\Sigma(s, s, Y) d W_{s}+\int_{r}^{t} \int_{r}^{v} \partial_{v} \Sigma\left(v, u,{ }_{n} Y\right)-\partial_{v} \Sigma(v, u, Y) d W_{u} d v
\end{aligned}
$$


for each $t \in[r, T]$ a.s. with a process ${ }_{n} \Delta \in \mathscr{C}\left([0, T], \mathbb{R}^{m}\right)$ satisfying

$$
{ }_{n} \Delta_{t}=\int_{r}^{r \vee t} \bar{B}\left(t, s,{ }_{n} Y\right)_{n} \dot{W}_{s}-R(t, s, Y) d s-\int_{r}^{r \vee t} \bar{B}(t, s, Y) d W_{s}
$$

for any $t \in[0, T]$ a.s. So, we let the terms ${ }_{n} Y_{r}-Y_{r}$ and ${ }_{n} \Delta$ unchanged, then for the constant $c_{p, 1}:=15^{p-1}(1+T-r)^{p}(T-r)^{p / 2-1}\left((T-r)^{p / 2}+\|h\|_{1,2, r}^{p}+w_{p}\right) \lambda^{p}$ we have

$$
\varphi_{n}(t)^{\frac{1}{p}} \leq \delta_{n, 1}^{\frac{1}{p}}+\delta_{n}(t)^{\frac{1}{p}}+\left(c_{p, 1} \int_{r}^{t_{n}} \delta_{n, 1}+\left(\delta_{n, 2}+\varepsilon_{n}+\varphi_{n}\right)(s) d s\right)^{\frac{1}{p}}
$$

for all $t \in[r, T]$, where we have set $\delta_{n, 1}:=E\left[\left\|_{n} Y^{r}-Y^{r}\right\|_{\infty}^{p}\right]$ and the measurable functions $\delta_{n}, \delta_{n, 2}, \varepsilon_{n}:[r, T] \rightarrow \mathbb{R}_{+}$are defined by

$$
\begin{aligned}
\delta_{n}(t) & :=E\left[\max _{j \in\left\{0, \ldots, k_{n}\right\}: t_{j, n} \leq t}\left|{ }_{n} \Delta_{t_{j, n}}\right|^{p}\right], \\
\delta_{n, 2}(s) & :=E\left[\left\|L_{n}\left({ }_{n} Y\right)^{\underline{s}_{n}}-{ }_{n} Y^{\underline{s}_{n}}\right\|_{\infty}^{p}+\left\|L_{n}(Y)^{\underline{s}_{n}}-Y^{\underline{s}_{n}}\right\|_{\infty}^{p}\right] \quad \text { and } \\
\varepsilon_{n}(s) & :=E\left[\|\|_{n} Y^{s}-{ }_{n} Y^{\underline{s}_{n}}\left\|_{\infty}^{p}+\right\| Y^{s}-Y^{\underline{s}_{n}} \|_{\infty}^{p}\right] .
\end{aligned}
$$

To obtain the estimate (4.5), we used the chain of inequalities: $E\left[\left\|L_{n}\left({ }_{n} Y\right)^{\underline{s}_{n}}-L_{n}(Y)^{\underline{s}_{n}}\right\|_{\infty}^{p}\right]$ $\leq E\left[\left.\left\|_{n} Y^{r}-Y^{r}\right\|_{\infty}^{p} \vee \max _{j \in\left\{0, \ldots, k_{n}\right\}: t_{j, n} \leq s}\right|_{n} Y_{t_{j, n}}-\left.Y_{t_{j, n}}\right|^{p}\right] \leq \delta_{n, 1}+\varphi_{n}(s)$, valid for every $s \in[r, T]$.

For the estimation of $\delta_{n}$ let us define two processes ${ }_{n, 3} \Delta,{ }_{n, 5} \Delta \in \mathscr{C}\left([0, T], \mathbb{R}^{m}\right)$ by ${ }_{n, 3} \Delta_{t}:=\int_{r}^{r \vee t} R\left(t, \underline{s}_{n},{ }_{n} Y\right)\left(\gamma_{n}(s)-1\right) d s$ and

$$
{ }_{n, 5} \Delta_{t}:=\int_{r}^{r \vee t}\left(\bar{B}\left(t, s,{ }_{n} Y\right)-\bar{B}\left(t, \underline{s}_{n},{ }_{n} Y\right)\right)_{n} \dot{W}_{s}-R\left(t, \underline{s}_{n},{ }_{n} Y\right) \gamma_{n}(s) d s
$$

and choose ${ }_{n, 4} \Delta \in \mathscr{C}\left([0, T], \mathbb{R}^{m}\right)$ such that ${ }_{n, 4} \Delta_{t}=\int_{r}^{r \vee t} \bar{B}\left(t, \underline{s}_{n},{ }_{n} Y\right) d\left({ }_{n} W_{s}-W_{s}\right)$ for any $t \in[0, T]$ a.s. Then ${ }_{n} \Delta$ admits the following representation:

$$
\begin{aligned}
{ }_{n} \Delta_{t}= & { }_{n, 3} \Delta_{t}+{ }_{n, 4} \Delta_{t}+{ }_{n, 5} \Delta_{t}+\int_{r}^{t} R\left(t, \underline{s}_{n},{ }_{n} Y\right)-R(t, s, Y) d s \\
& +\int_{r}^{t} \bar{B}\left(s, \underline{s}_{n},{ }_{n} Y\right)-\bar{B}(s, s, Y) d W_{s}+\int_{r}^{t} \int_{r}^{u} \partial_{u} \bar{B}\left(u, \underline{s}_{n},{ }_{n} Y\right)-\partial_{u} \bar{B}(u, s, Y) d W_{s} d u
\end{aligned}
$$

for all $t \in[r, T]$ a.s. Due to the assumptions, we may assume without loss of generality that the Lipschitz constant $\lambda$ is large enough such that

$$
|R(u, t, x)-R(u, s, y)| \leq \lambda d_{\infty}((t, x),(s, y))
$$

for any $s, t, u \in[r, T)$ with $s<t<u$ and every $x, y \in C\left([0, T], \mathbb{R}^{m}\right)$. Thus, for the constant $c_{p, 2}:=10^{p-1}(1+T-r)^{p}(T-r)^{p / 2-1}\left((T-r)^{p / 2}+w_{p}\right) \lambda^{p}$ we get that

$$
\begin{aligned}
\delta_{n}(t)^{\frac{1}{p}} \leq & \delta_{n, 3}(t)^{\frac{1}{p}}+\delta_{n, 4}(t)^{\frac{1}{p}}+\delta_{n, 5}(t)^{\frac{1}{p}} \\
& +\left(c_{p, 2} \int_{r}^{t_{n}} \delta_{n, 1}+\left(s-\underline{s}_{n}\right)^{\frac{p}{2}}+\left(\delta_{n, 2}+\varepsilon_{n}+\varphi_{n}\right)(s) d s\right)^{\frac{1}{p}}
\end{aligned}
$$

for every $t \in[r, T]$, where the increasing function $\delta_{n, i}:[r, T] \rightarrow \mathbb{R}_{+}$is given by

$$
\delta_{n, i}(t):=E\left[\max _{j \in\left\{0, \ldots, k_{n}\right\}: t_{j, n} \leq t}\left|{ }_{n, i} \Delta_{t_{j, n}}\right|^{p}\right] \text { for all } i \in\{3,4,5\}
$$


Thanks to Proposition 4.1 and Corollary 4.2, there are $\underline{c}_{p}, \bar{c}_{p}>0$ such that (4.1) and (4.4) hold when $c_{p}$ is replaced by $\underline{c}_{p}$ and $\bar{c}_{p}$, respectively. By combining (4.5) with (4.6), we see that

$$
\begin{aligned}
\varphi_{n}(t) \leq & c_{p, 4}\left|\mathbb{T}_{n}\right|^{\frac{p}{2}}\left(1+E\left[\left\|_{n} Y^{r}\right\|_{\infty}^{p}+\left\|Y^{r}\right\|_{\infty}^{p}\right]\right)+\left(5^{p-1}+c_{p, 3}(T-r)\right) \delta_{n, 1} \\
& +5^{p-1}\left(\delta_{n, 3}+\delta_{n, 4}+\delta_{n, 5}\right)(t)+c_{p, 3} \int_{r}^{t_{n}}\left(\delta_{n, 2}+\varphi_{n}\right)(s) d s
\end{aligned}
$$

for fixed $t \in[r, T]$, where $c_{p, 3}:=10^{p-1}\left(c_{p, 1}+c_{p, 2}\right)$ and $c_{p, 4}:=2^{p / 2}(T-r)\left(1+\underline{c}_{p}+\bar{c}_{p}\right) c_{p, 3}$. For this reason, Gronwall's inequality gives

$$
\varphi_{n}(t) / c_{p} \leq\left|\mathbb{T}_{n}\right|^{\frac{p}{2}}\left(1+E\left[\left\|_{n} Y^{r}\right\|_{\infty}^{p}+\left\|Y^{r}\right\|_{\infty}^{p}\right]\right)+\delta_{n, 1}+\sum_{i=2}^{5} \delta_{n, i}(t)
$$

with $c_{p}:=\exp \left(c_{p, 3}(T-r)\right)\left(5^{p-1}+c_{p, 4}\right)$, which implies the desired estimate.

By the estimate (3.2), Lemma 3.4 and Proposition 3.5, to prove (2.10), only the last remainder in the estimation of Proposition 4.3 should be investigated in more detail. Thus, let $\Phi_{h, n}:[r, T] \times C\left([0, T], \mathbb{R}^{m}\right) \times C\left([0, T], \mathbb{R}^{d}\right) \rightarrow \mathbb{R}^{m}$ be defined via

$$
\begin{aligned}
\Phi_{h, n}(s, y, w):= & B_{H}\left(\underline{s}_{n}, \underline{s}_{n}, y\right)\left(h(s)-h\left(\underline{s}_{n}\right)\right)+\bar{B}\left(\underline{s}_{n}, \underline{s}_{n}, y\right)\left(L_{n}(w)(s)-L_{n}(w)\left(\underline{s}_{n}\right)\right) \\
& +\Sigma\left(\underline{s}_{n}, \underline{s}_{n}, y\right)\left(w(s)-w\left(\underline{s}_{n}\right)\right)+\int_{\underline{s}_{n}}^{s} \int_{r}^{\underline{s}_{n}} \partial_{v} \bar{B}(v, u, y) d L_{n}(w)(u) d v
\end{aligned}
$$

for each $h \in W_{r}^{1,2}\left([0, T], \mathbb{R}^{d}\right)$ and any $n \in \mathbb{N}$. Whenever ${ }_{n} Y$ is a solution to (2.6), then we will utilize the following decomposition to deal with the considered remainder:

$$
\begin{aligned}
& \left(\bar{B}\left(t_{j, n}, s,{ }_{n} Y\right)-\bar{B}\left(t_{j, n}, \underline{s}_{n},{ }_{n} Y\right)\right)_{n} \dot{W}_{s}-R\left(t_{j, n}, \underline{s}_{n},{ }_{n} Y\right) \gamma_{n}(s) \\
& =\left(\bar{B}\left(t_{j, n}, s,{ }_{n} Y\right)-\bar{B}\left(t_{j, n}, \underline{s}_{n},{ }_{n} Y\right)-\partial_{x} \bar{B}\left(t_{j, n}, \underline{s}_{n},{ }_{n} Y\right)\left({ }_{n} Y_{s}-{ }_{n} Y_{\underline{s}_{n}}\right)\right)_{n} \dot{W}_{s} \\
& \quad+\partial_{x} \bar{B}\left(t_{j, n}, \underline{s}_{n},{ }_{n} Y\right)\left({ }_{n} Y_{s}-{ }_{n} Y_{\underline{s}_{n}}-\Phi_{h, n}\left(s,{ }_{n} Y, W\right)\right)_{n} \dot{W}_{s} \\
& \quad+\partial_{x} \bar{B}\left(t_{j, n}, \underline{s}_{n},{ }_{n} Y\right) \Phi_{h, n}\left(s,{ }_{n} Y, W\right)_{n} \dot{W}_{s}-R\left(t_{j, n}, \underline{s}_{n},{ }_{n} Y\right) \gamma_{n}(s)
\end{aligned}
$$

for all $j \in\left\{1, \ldots, k_{n}\right\}$ and each $s \in\left[r, t_{j, n}\right)$.

\subsection{Moment estimates for the first two remainders}

The first result in this section together with Lemma 3.2 provide an estimate for the first remainder appearing in (4.7).

Proposition 4.4. Let (C.4)-(C.6) be satisfied, $h \in W_{r}^{1,2}\left([0, T], \mathbb{R}^{d}\right)$ and $F$ be a product measurable functional on $[r, T] \times[r, T) \times C\left([0, T], \mathbb{R}^{m}\right)$ such that the following holds:

(i) There exists $\lambda \geq 0$ such that $|\bar{B}(u, t, x)-\bar{B}(u, s, x)|+\left|\partial_{u} \bar{B}(u, t, x)-\partial_{u} \bar{B}(u, s, x)\right|$ $\leq \lambda d_{\infty}((t, x),(s, x))$ for any $s, t, u \in[r, T)$ with $s<t<u$ and all $x \in C\left([0, T], \mathbb{R}^{m}\right)$.

(ii) The functional $[r, t) \times C\left([0, T], \mathbb{R}^{m}\right) \rightarrow \mathbb{R},(s, x) \mapsto F(t, s, x)$ is of class $\mathbb{C}^{1,2}$ for any $t \in(r, T]$ and there are $c_{0}, \eta, \lambda_{0} \geq 0$ such that

$$
\begin{aligned}
\left|\partial_{s} F(t, s, x)\right|+\left|\partial_{x x} F(t, s, x)\right| & \leq c_{0}\left(1+\|x\|_{\infty}^{\eta}\right) \quad \text { and } \\
\left|\partial_{x} F(u, t, x)-\partial_{x} F(u, s, x)\right| & \leq \lambda_{0} d_{\infty}((t, x),(s, x))
\end{aligned}
$$

for each $s, t, u \in[r, T)$ with $s<t<u$ and all $x \in C\left([0, T], \mathbb{R}^{m}\right)$. 
Then for any $p \geq 2$ there is $c_{p}>0$ such that for all $n \in \mathbb{N}$ and each solution ${ }_{n} Y$ to (2.6),

$$
\begin{gathered}
E\left[\max _{j \in\left\{1, \ldots, k_{n}\right\}} \int_{r}^{t_{j, n}} \mid F\left(t_{j, n}, s,{ }_{n} Y\right)\right. \\
\left.-F\left(t_{j, n}, \underline{s}_{n},{ }_{n} Y\right)-\left.\partial_{x} F\left(t_{j, n}, \underline{s}_{n},{ }_{n} Y\right)\left({ }_{n} Y_{s}-{ }_{n} Y_{\underline{s}_{n}}\right)\right|^{p} d s\right] \\
\leq c_{p}\left|\mathbb{T}_{n}\right|^{p-1}\left(1+E\left[\left\|_{n} Y^{r}\right\|_{\infty}^{(\eta \vee 2) p}\right]\right) .
\end{gathered}
$$

Proof. For any $j \in\left\{1, \ldots, k_{n}\right\}$ let the product measurable map ${ }_{n, j} \Delta:\left[r, t_{j, n}\right)^{2} \times \Omega \rightarrow \mathbb{R}^{1 \times m}$ be given by ${ }_{n, j} \Delta_{s, u}:=\partial_{x} F\left(t_{j, n}, u,{ }_{n} Y\right)-\partial_{x} F\left(t_{j, n}, \underline{s}_{n},{ }_{n} Y\right)$, if $u \in\left[\underline{s}_{n}, s\right]$, and ${ }_{n, j} \Delta_{s, u}:=0$, otherwise. Then from the functional Itô formula in [6] we infer that

$$
\begin{aligned}
& F\left(t_{j, n}, s,{ }_{n} Y\right)-F\left(t_{j, n}, \underline{s}_{n},{ }_{n} Y\right)-\partial_{x} F\left(t_{j, n}, \underline{s}_{n},{ }_{n} Y\right)\left({ }_{n} Y_{s}-{ }_{n} Y_{\underline{s}_{n}}\right) \\
& =\int_{\underline{s}_{n}}^{s} \partial_{u} F\left(t_{j, n}, u,{ }_{n} Y\right)+\frac{1}{2} \operatorname{tr}\left(\partial_{x x} F\left(t_{j, n}, u,{ }_{n} Y\right)\left(\Sigma \Sigma^{\prime}\right)\left(u, u,{ }_{n} Y\right)\right) d u \\
& \quad+\int_{\underline{s}_{n}}^{s} n, j \Delta_{s, u}\left(\underline{B}\left(u, u,{ }_{n} Y\right)+B_{H}\left(u, u,{ }_{n} Y\right) \dot{h}(u)+\bar{B}\left(u, u,{ }_{n} Y\right)_{n} \dot{W}_{u}\right) d u \\
& \quad+\int_{\underline{s}_{n}}^{s} n, j \Delta_{s, v} \int_{r}^{v} \partial_{v} \underline{B}\left(v, u,{ }_{n} Y\right)+\partial_{v} B_{H}\left(v, u,{ }_{n} Y\right) \dot{h}(u)+\partial_{v} \bar{B}\left(v, u,{ }_{n} Y\right)_{n} \dot{W}_{u} d u d v \\
& \quad+\int_{\underline{s}_{n}}^{s} n, j \Delta_{s, u} \Sigma\left(u, u,{ }_{n} Y\right) d W_{u}+\int_{\underline{s}_{n}}^{s}{ }_{n, j} \Delta_{s, v} \int_{r}^{v} \partial_{v} \Sigma\left(v, u,{ }_{n} Y\right) d W_{u} d v
\end{aligned}
$$

for each $s \in\left[r, t_{j, n}\right)$ a.s. Now, for $\eta_{0}:=\eta \vee 2$ Proposition 4.1 gives a constant $\underline{c}_{\eta_{0} p}>0$ such that (4.1) holds when $p$ and $c_{p}$ are replaced by $\eta_{0} p$ and $\underline{c}_{\eta_{0}}$, respectively. Then for the first two terms on the right-hand side in (4.8) we have

$$
\begin{aligned}
& E\left[\max _{j \in\left\{1, \ldots, k_{n}\right\}} \sup _{s \in\left[r, t_{j, n}\right)}\left|\int_{\underline{s}_{n}}^{s} \partial_{u} F\left(t_{j, n}, u,{ }_{n} Y\right)+\frac{1}{2} \operatorname{tr}\left(\partial_{x x} F\left(t_{j, n}, u,{ }_{n} Y\right)\left(\Sigma \Sigma^{\prime}\right)\left(u, u,{ }_{n} Y\right)\right) d u\right|^{p}\right] \\
& \leq 2^{p-1} c_{0}^{p}\left(s-\underline{s}_{n}\right)^{p} E\left[\left(1+\left\|_{n} Y\right\|_{\infty}^{\eta}\right)^{p}\right] \\
& \quad+2^{-1} c_{0}^{p}\left(s-\underline{s}_{n}\right)^{p-1} \int_{\underline{s}_{n}}^{s} E\left[\left(1+\left\|_{n} Y^{u}\right\|_{\infty}^{\eta}\right)^{p}\left|\left(\Sigma \Sigma^{\prime}\right)\left(u, u,{ }_{n} Y\right)\right|^{p}\right] d u \\
& \leq c_{p, 1}\left|\mathbb{T}_{n}\right|^{p}\left(1+E\left[\left\|_{n} Y^{r}\right\|_{\infty}^{\eta_{0} p}\right]\right)^{\frac{\eta}{\eta_{0}}}
\end{aligned}
$$

with $c_{p, 1}:=2^{2 p-1} c_{0}^{p}\left(2^{p}+c^{2 p}\right)\left(1+\underline{c}_{\eta_{0} p}\right)^{\eta / \eta_{0}}$. We note that $\left.\right|_{n, j} \Delta_{s, u} \mid \leq \lambda_{0} d_{\infty}\left(\left(s,{ }_{n} Y\right),\left(\underline{s}_{n},{ }_{n} Y\right)\right)$ for each $j \in\left\{1, \ldots, k_{n}\right\}$ and all $s, u \in\left[r, t_{j, n}\right)$ and by setting $\bar{c}_{p}:=2^{3 p / 2} \lambda_{0}^{p}\left(1+\underline{c}_{\eta_{0} p}\right)^{1 / \eta_{0}}$, we obtain that

$$
\lambda_{0}^{p} E\left[d_{\infty}\left(\left(s,{ }_{n} Y\right),\left(\underline{s}_{n},{ }_{n} Y\right)\right)^{2 p}\right]^{\frac{1}{2}} \leq \bar{c}_{p}\left|\mathbb{T}_{n}\right|^{\frac{p}{2}}\left(1+E\left[\left\|_{n} Y^{r}\right\|_{\infty}^{\eta_{0} p}\right]\right)^{\frac{1}{\eta_{0}}}
$$

for each $s \in[r, T]$. Consequently, the Cauchy-Schwarz inequality gives us the following bound for the third and the sixth expression in the decomposition (4.8):

$$
\begin{aligned}
& E\left[\max _{j \in\left\{1, \ldots, k_{n}\right\}} \int_{r}^{t_{j, n}}\left|\int_{\underline{s}_{n}}^{s}{ }_{n, j} \Delta_{s, v}\left(\underline{B}\left(v, v,{ }_{n} Y\right)+\int_{r}^{v} \partial_{v} \underline{B}\left(v, u,{ }_{n} Y\right) d u\right) d v\right|^{p} d s\right] \\
& \leq 2^{p-1} c^{p} \int_{r}^{T}\left(s-\underline{s}_{n}\right)^{p} \lambda_{0}^{p} E\left[d_{\infty}\left(\left(s,{ }_{n} Y\right),\left(\underline{s}_{n},{ }_{n} Y\right)\right)^{p}\left(1+\left\|{ }_{n} Y^{s}\right\|_{\infty}^{\kappa}\right)^{p}\right] d s \\
& \quad+2^{p-1} c^{p} \int_{r}^{T} \lambda_{0}^{p} E\left[d_{\infty}\left(\left(s,{ }_{n} Y\right),\left(\underline{s}_{n},{ }_{n} Y\right)\right)^{p}\left(s-\underline{s}_{n}\right)^{p-1} \int_{\underline{s}_{n}}^{s}\left(\int_{r}^{v} 1+\left\|_{n} Y^{u}\right\|_{\infty}^{\kappa} d u\right)^{p} d v\right] d s \\
& \leq c_{p, 2}\left|\mathbb{T}_{n}\right|^{p}\left(1+E\left[\left\|_{n} Y^{r}\right\|_{\infty}^{\eta_{0} p}\right]\right)^{\frac{2}{\eta_{0}}} \int_{r}^{T}\left(s-\underline{s}_{n}\right)^{\frac{p}{2}} d s
\end{aligned}
$$


for $c_{p, 2}:=2^{5 p / 2-1}\left(1+(T-r)^{p}\right) c^{p}\left(1+\underline{c}_{\eta_{0} p}\right)^{1 / \eta_{0}} \bar{c}_{p}$. For the fourth expression we apply the Cauchy-Schwarz inequality twice, which entails that

$$
\begin{aligned}
& E\left[\max _{j \in\left\{1, \ldots, k_{n}\right\}} \int_{r}^{t_{j, n}}\left|\int_{\underline{s}_{n}}^{s}{ }_{n, j} \Delta_{s, u} B_{H}\left(u, u,{ }_{n} Y\right) d h(u)\right|^{p} d s\right] \\
& \leq\|h\|_{1,2, r}^{p} c^{p} \int_{r}^{T}\left(s-\underline{s}_{n}\right)^{\frac{p}{2}} \lambda_{0}^{p} E\left[d_{\infty}\left(\left(s,{ }_{n} Y\right),\left(\underline{s}_{n},{ }_{n} Y\right)\right)^{p}\left(1+\left\|_{n} Y\right\|_{\infty}^{\kappa}\right)^{p}\right] d s \\
& \leq c_{p, 3}\left|\mathbb{T}_{n}\right|^{p}\left(1+E\left[\left\|_{n} Y^{r}\right\|_{\infty}^{\eta_{0} p}\right]\right)^{\frac{2}{\eta_{0}}},
\end{aligned}
$$

where $c_{p, 3}:=2^{3 p / 2}(T-r)\|h\|_{1,2, r}^{p} c^{p}\left(1+\underline{c}_{\eta_{0}}\right)^{1 / \eta_{0}} \bar{c}_{p}$. Proceeding similarly, it follows for the seventh expression that

$$
\begin{aligned}
& E\left[\max _{j \in\left\{1, \ldots, k_{n}\right\}} \int_{r}^{t_{j, n}}\left|\int_{\underline{s}_{n}}^{s}{ }_{n, j} \Delta_{s, v} \int_{r}^{v} \partial_{v} B_{H}\left(v, u,{ }_{n} Y\right) d h(u) d v\right|^{p} d s\right] \\
& \leq \int_{r}^{T}\left(s-\underline{s}_{n}\right)^{p-1} \int_{\underline{s}_{n}}^{s} \lambda_{0}^{p} E\left[d_{\infty}\left(\left(s,{ }_{n} Y\right),\left(\underline{s}_{n},{ }_{n} Y\right)\right)^{p}\left|\int_{r}^{v} \partial_{v} B_{H}\left(v, u,{ }_{n} Y\right) d h(u)\right|^{p}\right] d v d s \\
& \leq c_{p, 4}\left|\mathbb{T}_{n}\right|^{p}\left(1+E\left[\left\|_{n} Y^{r}\right\|_{\infty}^{\eta_{0} p}\right]\right)^{\frac{2}{\eta_{0}}} \int_{r}^{T}\left(s-\underline{s}_{n}\right)^{\frac{p}{2}} d s
\end{aligned}
$$

with $c_{p, 4}:=(T-r)^{p / 2-1} c_{p, 3}$. We turn to the fifth and eight term in (4.8) and once again apply the Cauchy-Schwarz inequality, which leads us to

$$
\begin{aligned}
& E\left[\max _{j \in\left\{1, \ldots, k_{n}\right\}} \int_{r}^{t_{j, n}}\left|\int_{\underline{s}_{n}}^{s}{ }_{n, j} \Delta_{s, v}\left(\bar{B}\left(v, v,{ }_{n} Y\right)_{n} \dot{W}_{v}+\int_{r}^{v} \partial_{v} \bar{B}\left(v, u,{ }_{n} Y\right) d_{n} W_{u}\right) d v\right|^{p} d s\right] \\
& \leq 2^{p-1} c^{p} \int_{r}^{T}\left(s-\underline{s}_{n}\right)^{\frac{p}{2}} \lambda_{0}^{p} E\left[d_{\infty}\left(\left(s,{ }_{n} Y\right),\left(\underline{s}_{n},{ }_{n} Y\right)\right)^{p}\left(\left.\left.\int_{\underline{s}_{n}}^{s}\right|_{n} \dot{W}_{v}\right|^{2} d v\right)^{\frac{p}{2}}\right] d s \\
& \quad+2^{p-1} c^{p} \int_{r}^{T}\left(s-\underline{s}_{n}\right)^{p-1} \int_{\underline{s}_{n}}^{s} \lambda_{0}^{p} E\left[d_{\infty}\left(\left(s,{ }_{n} Y\right),\left(\underline{s}_{n},{ }_{n} Y\right)\right)^{p}\left(\int_{r}^{v}\left|{ }_{n} \dot{W}_{u}\right| d u\right)^{p}\right] d v d s \\
& \leq c_{p, 5}\left|\mathbb{T}_{n}\right|^{p}\left(1+E\left[\left\|_{n} Y^{r}\right\|_{\infty}^{\eta_{0} p}\right]\right)^{\frac{1}{\eta_{0}}}
\end{aligned}
$$

for $c_{p, 5}:=2^{2 p-1} \hat{w}_{p, 2}^{1 / 2}(T-r)\left(1+(T-r)^{p} /(p+1)\right) c^{p} \bar{c}_{p}$. By using the constant $\bar{c}_{\mathbb{T}}$ appearing in condition (C.4), we derive the following estimate for the ninth term:

$$
\begin{aligned}
E\left[\max _{j \in\left\{1, \ldots, k_{n}\right\}}\right. & \left.\int_{r}^{t_{j, n}}\left|\int_{\underline{s}_{n}}^{s} n_{j} \Delta_{s, u} \Sigma\left(u, u,{ }_{n} Y\right) d W_{u}\right|^{p} d s\right] \\
& \leq w_{p} c^{p} \sum_{j=1}^{k_{n}} \int_{r}^{t_{j, n}}\left(s-\underline{s}_{n}\right)^{\frac{p}{2}-1} \int_{\underline{s}_{n}}^{s} \lambda_{0}^{p} E\left[d_{\infty}\left(\left(s,{ }_{n} Y\right),\left(\underline{s}_{n},{ }_{n} Y\right)\right)^{p}\right] d u d s \\
& \leq c_{p, 6}\left|\mathbb{T}_{n}\right|^{p-1}\left(1+E\left[\left\|_{n} Y^{r}\right\|_{\infty}^{\eta_{\infty} p}\right]\right)^{\frac{1}{\eta_{0}}},
\end{aligned}
$$

where $c_{p, 6}:=2^{p / 2} w_{p} c^{p} \bar{c}_{p}(T-r) \bar{c}_{\mathbb{T}}$. Finally, for the last expression we now readily estimate that

$$
\begin{aligned}
& E\left[\max _{j \in\left\{1, \ldots, k_{n}\right\}} \int_{r}^{t_{j, n}}\left|\int_{\underline{s}_{n}}^{s}{ }_{n, j} \Delta_{s, v} \int_{r}^{v} \partial_{v} \Sigma\left(v, u,{ }_{n} Y\right) d W_{u} d v\right|^{p} d s\right] \\
& \leq \int_{r}^{T}\left(s-\underline{s}_{n}\right)^{p-1} \int_{\underline{s}_{n}}^{s} E\left[\lambda_{0}^{p} d_{\infty}\left(\left(s,{ }_{n} Y\right),\left(\underline{s}_{n},{ }_{n} Y\right)\right)^{p}\left|\int_{r}^{v} \partial_{v} \Sigma\left(v, u,{ }_{n} Y\right) d W_{u}\right|^{p}\right] d v d s \\
& \leq c_{p, 7}\left|\mathbb{T}_{n}\right|^{p}\left(1+E\left[\|\|_{n} Y^{r} \|_{\infty}^{\eta_{0} p}\right]\right)^{\frac{1}{\eta_{0}}} \int_{r}^{T}\left(s-\underline{s}_{n}\right)^{\frac{p}{2}} d s
\end{aligned}
$$


for $c_{p, 7}:=2^{p / 2} c^{p} \bar{c}_{p} w_{2 p}^{1 / 2}(T-r)^{p / 2}$. So, we let $c_{p, 8}:=(T-r)\left((T-r) c_{p, 1}+c_{p, 3}+c_{p, 5}\right)$ and $c_{p, 9}:=(T-r)^{p / 2+2}\left(c_{p, 2}+c_{p, 4}+c_{p, 7}\right)$ and conclude by setting $c_{p}:=7^{p-1}\left(c_{p, 6}+c_{p, 8}+c_{p, 9}\right)$.

Next, we give a bound for the second remainder in (4.7), which allows for another application of Lemma 3.2, according to Remark 3.3.

Lemma 4.5. Let (C.5)-(C.7) be valid and $h \in W_{r}^{1,2}\left([0, T], \mathbb{R}^{d}\right)$. Then for each $p \geq 2$ there is $c_{p}>0$ such that each $n \in \mathbb{N}$ and any solution ${ }_{n} Y$ to (2.6) satisfy

$$
E\left[\left|{ }_{n} Y_{s}-{ }_{n} Y_{\underline{s}_{n}}-\Phi_{h, n}\left(s,{ }_{n} Y, W\right)\right|^{p}\right] \leq c_{p}\left|\mathbb{T}_{n}\right|^{p}\left(1+E\left[\left\|_{n} Y^{r}\right\|_{\infty}^{2 p}\right]\right)^{\frac{1}{2}}
$$

for every $s \in[r, T)$.

Proof. From Fubini's theorem for both deterministic and stochastic integrals and the definition of $\Phi_{h, n}$ we get that

$$
\begin{aligned}
& { }_{n} Y_{s}-{ }_{n} Y_{\underline{s}_{n}}-\Phi_{h, n}\left(s,{ }_{n} Y, W\right)=\int_{\underline{s}_{n}}^{s} \underline{B}\left(u, u,{ }_{n} Y\right) d u \\
& +\int_{\underline{s}_{n}}^{s} B_{H}\left(u, u,{ }_{n} Y\right)-B_{H}\left(\underline{s}_{n}, \underline{s}_{n},{ }_{n} Y\right) d h(u)+\int_{\underline{s}_{n}}^{s} \bar{B}\left(u, u,{ }_{n} Y\right)-\bar{B}\left(\underline{s}_{n}, \underline{s}_{n},{ }_{n} Y\right) d_{n} W_{u} \\
& +\int_{\underline{s}_{n}}^{s} \int_{r}^{v} \partial_{v} \underline{B}\left(v, u,{ }_{n} Y\right) d u d v+\int_{\underline{s}_{n}}^{s} \int_{r}^{v} \partial_{v} B_{H}\left(v, u,{ }_{n} Y\right) d h(u) d v \\
& +\int_{\underline{s}_{n}}^{s} \int_{\underline{s}_{n}}^{v} \partial_{v} \bar{B}\left(v, u,{ }_{n} Y\right) d_{n} W_{u} d v+\int_{\underline{s}_{n}}^{s} \Sigma\left(u, u,{ }_{n} Y\right)-\Sigma\left(\underline{s}_{n}, \underline{s}_{n},{ }_{n} Y\right) d W_{u} \\
& +\int_{\underline{s}_{n}}^{s} \int_{r}^{v} \partial_{v} \Sigma\left(v, u,{ }_{n} Y\right) d W_{u} d v \quad \text { a.s. }
\end{aligned}
$$

Proposition 4.1 provides a constant $\underline{c}_{2 p}>0$ such that (4.1) holds when $p$ and $c_{p}$ are replaced by $2 p$ and $\underline{c}_{2 p}$, respectively. We set $\bar{c}_{p, 1}:=\left(1+\underline{c}_{2 p}\right)^{1 / 2}$ and $\bar{c}_{p, 2}:=\lambda^{p}+(T-r)^{p / 2} c^{p}$ and define eight constants as follows:

$$
\begin{aligned}
& c_{p, 1}:=2^{2 p} c^{p} \bar{c}_{p, 1}, \quad c_{p, 2}:=2^{3 p}\|h\|_{1,2, r}^{p} \bar{c}_{p, 1} \bar{c}_{p, 2}, \quad c_{p, 3}:=2^{\frac{3}{2} p} 3^{p} \hat{w}_{p, 2}^{\frac{1}{2}} \bar{c}_{p, 1} \bar{c}_{p, 2}, \\
& c_{p, 4}:=(T-r)^{p} c_{p, 1}, \quad c_{p, 5}:=2^{2 p}(T-r)^{\frac{p}{2}}\|h\|_{1,2, r}^{p} c^{p} \bar{c}_{p, 1}, \quad c_{p, 6}:=2^{\frac{3}{2} p} \hat{w}_{p, 1}(T-r)^{\frac{p}{2}} c^{p}, \\
& c_{p, 7}:=2^{p} 3^{p} w_{p} \bar{c}_{p, 1} \bar{c}_{p, 2} \quad \text { and } \quad c_{p, 8}:=2^{p} w_{p}(T-r)^{\frac{p}{2}} c^{p} .
\end{aligned}
$$

By using the inequalities of Jensen and Cauchy-Schwarz, (3.3) and (3.4), it follows readily that the $p$-th moment of the $i$-th integral in the decomposition (4.9) is bounded by $c_{p, i}\left|\mathbb{T}_{n}\right|^{p}\left(1+E\left[\left\|_{n} Y^{r}\right\|_{\infty}^{2 p}\right]\right)^{1 / 2}$ for all $i \in\{1, \ldots, 8\}$. We set $c_{p}:=8^{p-1}\left(c_{p, 1}+\cdots+c_{p, 8}\right)$ and the asserted estimate follows.

\subsection{A second moment estimate for the third remainder}

We bound the third remainder in (4.7) by repeatedly using an estimate that follows for any $n \in \mathbb{N}$ with $k_{n} \geq 2$ from Doob's $L^{2}$-maximal inequality; see [7][Lemma 33] for details.

(v) For every $l \in\{1, \ldots, d\}$ assume that $\left({ }_{l} U_{i}\right)_{i \in\left\{1, \ldots, k_{n}-1\right\}}$ and $\left({ }_{l} V_{i}\right)_{i \in\left\{1, \ldots, k_{n}-1\right\}}$ are two sequences of $\mathbb{R}^{1 \times m}$-valued and $\mathbb{R}^{m}$-valued random vectors, respectively, such that ${ }_{l} U_{i}$ is $\mathscr{F}_{t_{i-1, n}}$-measurable, ${ }_{l} V_{i}$ is $\mathscr{F}_{t_{i}, n}$-measurable,

$$
E\left[\left.{ }_{l} U_{i}\right|^{4}+\left.{ }_{l} V_{i}\right|^{4}\right]<\infty \quad \text { and } E\left[{ }_{l} V_{i} \mid \mathscr{F}_{t_{i-1, n}}\right]=0 \text { a.s. }
$$

for all $i \in\left\{1, \ldots, k_{n}-1\right\}$. Then

$$
E\left[\max _{j \in\left\{1, \ldots, k_{n}\right\}}\left|\sum_{i=1}^{j-1} \sum_{l=1}^{d}{ }_{l} U_{i l} V_{i}\right|^{2}\right] \leq 4 \sum_{i=1}^{k_{n}-1} \sum_{l_{1}, l_{2}=1}^{d} E\left[{ }_{l_{1}} U_{i l_{1}} V_{i l_{2}} V_{i}^{\prime} l_{2} U_{i}^{\prime}\right] .
$$


Proposition 4.6. Let (C.5)-(C.8) be satisfied and $h \in W_{r}^{1,2}\left([0, T], \mathbb{R}^{d}\right)$. Then there is $c_{2}>0$ such that for each $n \in \mathbb{N}$ and any solution ${ }_{n} Y$ to (2.6) it holds that

$$
\begin{gathered}
E\left[\max _{j \in\left\{0 \ldots, k_{n}\right\}} \mid \int_{r}^{t_{j, n}} \partial_{x} \bar{B}\left(t_{j, n}, \underline{s}_{n},{ }_{n} Y\right)\right. \\
\left.\Phi_{h, n}\left(s,{ }_{n} Y, W\right)_{n} \dot{W}_{s}-\left.R\left(t_{j, n}, \underline{s}_{n},{ }_{n} Y\right) \gamma_{n}(s) d s\right|^{2}\right] \\
\leq c_{2}\left|\mathbb{T}_{n}\right|\left(1+E\left[\left\|_{n} Y^{r}\right\|_{\infty}^{2}\right]\right) .
\end{gathered}
$$

Proof. By the definition (2.8) of the mapping $R$, we can write the $k$-th coordinate of $\partial_{x} \bar{B}\left(t_{j, n}, \underline{s}_{n},{ }_{n} Y\right) \Phi_{h, n}\left(s,{ }_{n} Y, W\right)_{n} \dot{W}_{s}-R\left(t_{j, n}, \underline{s}_{n},{ }_{n} Y\right) \gamma_{n}(s)$ in the form

$$
\sum_{l=1}^{d} \partial_{x} \bar{B}_{k, l}\left(t_{j, n}, \underline{s}_{n},{ }_{n} Y\right)\left(\Phi_{h, n}\left(s,{ }_{n} Y, W\right)_{n} \dot{W}_{s}^{(l)}-((1 / 2) \bar{B}+\Sigma)\left(\underline{s}_{n}, \underline{s}_{n},{ }_{n} Y\right) \gamma_{n}(s) e_{l}\right)
$$

for each $j \in\left\{1, \ldots, k_{n}\right\}$, any $k \in\{1, \ldots, m\}$ and all $s \in\left[r, t_{j, n}\right)$, where $X^{(l)}$ stands for the $l$-th coordinate of any $\mathbb{R}^{d}$-valued process $X$ for each $l \in\{1, \ldots, d\}$. Based on this identity, we use the following decomposition:

$$
\begin{aligned}
\Phi_{h, n}\left(s,{ }_{n} Y, W\right)_{n} \dot{W}_{s}^{(l)}-((1 / 2) \bar{B}+\Sigma)\left(\underline{s}_{n}, \underline{s}_{n},{ }_{n} Y\right) \gamma_{n}(s) e_{l} \\
=B_{H}\left(\underline{s}_{n}, \underline{s}_{n},{ }_{n} Y\right)\left(h\left(s_{n}\right)-h\left(\underline{s}_{n}\right)\right)_{n} \dot{W}_{s}^{(l)}+\bar{B}\left(\underline{s}_{n}, \underline{s}_{n},{ }_{n} Y\right)\left({ }_{n} W_{s_{n}}-{ }_{n} W_{\underline{s}_{n}}\right)_{n} \dot{W}_{s}^{(l)} \\
\quad+\Sigma\left(\underline{s}_{n}, \underline{s}_{n},{ }_{n} Y\right)\left(\Delta W_{s_{n} n} \dot{W}_{s}^{(l)}-\gamma_{n}(s) e_{l}\right)+B B_{H}\left(\underline{s}_{n}, \underline{s}_{n},{ }_{n} Y\right)\left(h(s)-h\left(s_{n}\right)\right)_{n} \dot{W}_{s}^{(l)} \\
\quad+\bar{B}\left(\underline{s}_{n}, \underline{s}_{n},{ }_{n} Y\right)\left(\left({ }_{n} W_{s}-{ }_{n} W_{s_{n}}\right)_{n} \dot{W}_{s}^{(l)}-(1 / 2) \gamma_{n}(s) e_{l}\right) \\
\quad+\Sigma\left(\underline{s}_{n}, \underline{s}_{n},{ }_{n} Y\right)\left(W_{s}-W_{s_{n}}\right)_{n} \dot{W}_{s}^{(l)}+\int_{\underline{s}_{n}}^{s} \int_{r}^{\underline{s}_{n}} \partial_{v} \bar{B}\left(v, u,{ }_{n} Y\right) d_{n} W_{u} d v{ }_{n} \dot{W}_{s}^{(l)}
\end{aligned}
$$

with $l \in\{1, \ldots, d\}$. To handle the first appearing term, we decompose the integral and apply Fubini's theorem for stochastic integrals to rewrite that

$$
\begin{aligned}
& \int_{r}^{t_{j, n}} \partial_{x} \bar{B}_{k, l}\left(t_{j, n}, \underline{s}_{n},{ }_{n} Y\right) B_{H}\left(\underline{s}_{n}, \underline{s}_{n},{ }_{n} Y\right)\left(h\left(s_{n}\right)-h\left(\underline{s}_{n}\right)\right) d_{n} W_{s}^{(l)} \\
& =\int_{r}^{t_{j-1, n}} \partial_{x} \bar{B}_{k, l}\left(s, s_{n},{ }_{n} Y\right) B_{H}\left(s_{n}, s_{n},{ }_{n} Y\right)\left(h\left(\bar{s}_{n}\right)-h\left(s_{n}\right)\right) d W_{s}^{(l)} \\
& \quad+\int_{r}^{t_{j, n}} \int_{r}^{t \wedge t_{j-1, n}} \partial_{t} \partial_{x} \bar{B}_{k, l}\left(t, s_{n},{ }_{n} Y\right) B_{H}\left(s_{n}, s_{n},{ }_{n} Y\right)\left(h\left(\bar{s}_{n}\right)-h\left(s_{n}\right)\right) d W_{s}^{(l)} d t \quad \text { a.s. }
\end{aligned}
$$

for any $j \in\left\{1, \ldots, k_{n}\right\}$, every $k \in\{1, \ldots, m\}$ and each $l \in\{1, \ldots, d\}$. By Proposition 4.1, there is $\underline{c}_{2}>0$ such that (4.1) holds for $p=2$ with $\underline{c}_{2}$ instead of $c_{p}$. Therefore,

$$
\begin{aligned}
& E\left[\max _{j \in\left\{0, \ldots, k_{n}\right\}} \sum_{k=1}^{m}\left|\sum_{l=1}^{d} \int_{r}^{t_{j, n}} \partial_{x} \bar{B}_{k, l}\left(t_{j, n}, \underline{s}_{n},{ }_{n} Y\right) B_{H}\left(\underline{s}_{n}, \underline{s}_{n},{ }_{n} Y\right)\left(h\left(s_{n}\right)-h\left(\underline{s}_{n}\right)\right) d_{n} W_{s}^{(l)}\right|^{2}\right] \\
& \leq 2 w_{2} c^{2} \bar{c}^{2} \int_{r}^{T} E\left[\left(1+\left\|_{n} Y^{s_{n}}\right\|_{\infty}^{\kappa}\right)^{2}\right]\left|h\left(\bar{s}_{n}\right)-h\left(s_{n}\right)\right|^{2} d s \\
& \quad+2 w_{2}(T-r) c^{2} \bar{c}^{2} \int_{r}^{T} \int_{r}^{t} E\left[\left(1+\left\|_{n} Y^{s_{n}}\right\|_{\infty}^{\kappa}\right)^{2}\right]\left|h\left(\bar{s}_{n}\right)-h\left(s_{n}\right)\right|^{2} d s d t \\
& \leq c_{2,1}\left|\mathbb{T}_{n}\right|\left(1+E\left[\left\|_{n} Y^{r}\right\|_{\infty}^{2}\right]\right)^{\kappa}
\end{aligned}
$$

with $c_{2,1}:=2^{3} w_{2}\left(1+(T-r)^{2} / 2\right)(T-r)\|h\|_{1,2, r}^{2} c^{2} \bar{c}^{2}\left(1+\underline{c}_{2}\right)^{\kappa}$. Proceeding similarly, we obtain for the second term in the decomposition (4.11) that

$$
\int_{r}^{t_{j, n}} \partial_{x} \bar{B}_{k, l}\left(t_{j, n}, \underline{s}_{n},{ }_{n} Y\right) \bar{B}\left(\underline{s}_{n}, \underline{s}_{n},{ }_{n} Y\right)\left({ }_{n} W_{s_{n}}-{ }_{n} W_{\underline{s}_{n}}\right) d_{n} W_{s}^{(l)}
$$


Support characterization for stochastic Volterra integral equations

$$
\begin{aligned}
= & \int_{r}^{t_{j-1, n}} \partial_{x} \bar{B}_{k, l}\left(s, s_{n},{ }_{n} Y\right) \bar{B}\left(s_{n}, s_{n},{ }_{n} Y\right) \Delta W_{s_{n}} d W_{s}^{(l)} \\
& +\int_{r}^{t_{j, n}} \int_{r}^{t \wedge t_{j-1, n}} \partial_{t} \partial_{x} \bar{B}_{k, l}\left(t, s_{n},{ }_{n} Y\right) \bar{B}\left(s_{n}, s_{n},{ }_{n} Y\right) \Delta W_{s_{n}} d W_{s}^{(l)} d t \quad \text { a.s. }
\end{aligned}
$$

for every $j \in\left\{1, \ldots, k_{n}\right\}$, each $k \in\{1, \ldots, m\}$ and any $l \in\{1, \ldots, d\}$. Hence, by setting $c_{2,2}:=2 w_{2}\left(1+(T-r)^{2} / 2\right)(T-r) d c^{2} \bar{c}^{2}$, it follows readily that

$$
\begin{aligned}
& E\left[\max _{j \in\left\{0, \ldots, k_{n}\right\}} \sum_{k=1}^{m}\left|\sum_{l=1}^{d} \int_{r}^{t_{j, n}} \partial_{x} \bar{B}_{k, l}\left(t_{j, n}, \underline{s}_{n},{ }_{n} Y\right) \bar{B}\left(\underline{s}_{n}, \underline{s}_{n},{ }_{n} Y\right)\left({ }_{n} W_{s_{n}}-{ }_{n} W_{\underline{s}_{n}}\right) d_{n} W_{s}^{(l)}\right|^{2}\right] \\
& \leq 2 w_{2} c^{2} \bar{c}^{2} \int_{r}^{T}\left(E\left[\left|\Delta W_{t_{n}}\right|^{2}\right]+(T-r) \int_{r}^{t} E\left[\left|\Delta W_{s_{n}}\right|^{2}\right] d s\right) d t \leq c_{2,2}\left|\mathbb{T}_{n}\right| .
\end{aligned}
$$

To deal with the third expression in (4.11), we utilize the $\mathbb{R}^{d}$-valued $\mathscr{F}_{i, n}$-measurable random vector

$$
{ }_{l, n} V_{i}:=\Delta W_{t_{i, n}} \Delta W_{t_{i, n}}^{(l)}-\Delta t_{i, n} e_{l},
$$

which is independent of $\mathscr{F}_{t_{i-1, n}}$ and satisfies $E\left[l_{, n} V_{i}\right]=0$ for any $i \in\left\{1, \ldots, k_{n}\right\}$ and each $l \in\{1, \ldots, d\}$. We note that if $\mathbb{I}_{l_{2}, l_{1}} \in \mathbb{R}^{d \times d}$ denotes the matrix whose $\left(l_{2}, l_{1}\right)$-entry is 1 and whose all other entries are zero, then

$$
E\left[l_{1, n} V_{i l_{2}, n} V_{i}^{\prime}\right]=\left(\Delta t_{i, n}\right)^{2}\left(\mathbb{1}_{\left\{l_{2}\right\}}\left(l_{1}\right) \mathbb{I}_{d}+\mathbb{I}_{l_{2}, l_{1}}\right)
$$

whenever $i \in\left\{1, \ldots, k_{n}\right\}$ and $l_{1}, l_{2} \in\{1, \ldots, d\}$. Now, by decomposing the integral once again, we obtain that

$$
\begin{aligned}
& \int_{r}^{t_{j, n}} \partial_{x} \bar{B}_{k, l}\left(t_{j, n}, \underline{s}_{n},{ }_{n} Y\right) \Sigma\left(\underline{s}_{n}, \underline{s}_{n},{ }_{n} Y\right)\left(\Delta W_{s_{n} n} \dot{W}_{s}^{(l)}-\gamma_{n}(s) e_{l}\right) d s \\
& =\sum_{i=1}^{j-1} \partial_{x} \bar{B}_{k, l}\left(t_{i, n}, t_{i-1, n},{ }_{n} Y\right) \Sigma\left(t_{i-1, n}, t_{i-1, n},{ }_{n} Y\right)_{l, n} V_{i} \\
& \quad+\sum_{i_{2}=1}^{j-1} \int_{t_{i_{2}, n}}^{t_{i_{2}+1, n}} \sum_{i_{1}=1}^{i_{2}} \partial_{t} \partial_{x} \bar{B}_{k, l}\left(t, t_{i_{1}-1, n},{ }_{n} Y\right) \Sigma\left(t_{i_{1}-1, n}, t_{i_{1}-1, n},{ }_{n} Y\right)_{l, n} V_{i_{1}} d t \\
& =\sum_{i=1}^{j-1} \partial_{x} \bar{B}_{k, l}\left(t_{i, n}, t_{i-1, n},{ }_{n} Y\right) \Sigma\left(t_{i-1, n}, t_{i-1, n},{ }_{n} Y\right)_{l, n} V_{i} \\
& \quad+\int_{t_{1, n}}^{t_{j, n}} \sum_{i=1}^{i_{n}(t)} \partial_{t} \partial_{x} \bar{B}_{k, l}\left(t, t_{i-1, n},{ }_{n} Y\right) \Sigma\left(t_{i-1, n}, t_{i-1, n},{ }_{n} Y\right)_{l, n} V_{i} d t
\end{aligned}
$$

for all $j \in\left\{1, \ldots, k_{n}\right\}$, each $k \in\{1, \ldots, m\}$ and every $l \in\{1, \ldots, d\}$ with the function $i_{n}:[r, T] \rightarrow\left\{0, \ldots, k_{n}\right\}$ given by

$$
i_{n}(t):=\max \left\{i \in\left\{0, \ldots, k_{n}\right\} \mid t_{i, n} \leq t\right\},
$$

which satisfies $i_{n}=i$ on $\left[t_{i, n}, t_{i+1, n}\right)$ for any $i \in\left\{0, \ldots, k_{n}-1\right\}$ and $i_{n}(T)=k_{n}$. For this reason, the estimate (4.10) gives us that

$$
\begin{aligned}
& E\left[\max _{j \in\left\{0, \ldots, k_{n}\right\}} \sum_{k=1}^{m}\left|\int_{r}^{t_{j, n}} \sum_{l=1}^{d} \partial_{x} \bar{B}_{k, l}\left(t_{j, n}, \underline{s}_{n},,_{n} Y\right) \Sigma\left(\underline{s}_{n}, \underline{s}_{n},{ }_{n} Y\right)\left(\Delta W_{s_{n} n} \dot{W}_{s}^{(l)}-\gamma_{n}(s) e_{l}\right) d s\right|^{2}\right] \\
& \leq 2^{4}\left|\mathbb{T}_{n}\right| \sum_{i=1}^{k_{n}-1} \Delta t_{i, n} \sum_{k=1}^{m} \sum_{l=1}^{d} E\left[\left|\partial_{x} \bar{B}_{k, l}\left(t_{i, n}, t_{i-1, n},{ }_{n} Y\right) \Sigma\left(t_{i-1, n}, t_{i-1, n},{ }_{n} Y\right)\right|^{2}\right]
\end{aligned}
$$


Support characterization for stochastic Volterra integral equations

$$
\begin{aligned}
& +2(T-r) \int_{t_{1, n}}^{T} \sum_{k=1}^{m} E\left[\left|\sum_{i=1}^{i_{n}(t)} \sum_{l=1}^{d} \partial_{t} \partial_{x} \bar{B}_{k, l}\left(t, t_{i-1, n},{ }_{n} Y\right) \Sigma\left(t_{i-1, n}, t_{i-1, n},{ }_{n} Y\right)_{l, n} V_{i}\right|^{2}\right] d t \\
\leq & c_{2,3}\left|\mathbb{T}_{n}\right|
\end{aligned}
$$

with $c_{2,3}:=2^{4}\left(1+(T-r)^{2}\right)(T-r) c^{2} \bar{c}^{2}$, since $\bar{x}^{\prime} \mathbb{I}_{l_{2}, l_{1}} \bar{y} \leq(1 / 2)\left(\bar{x}_{l_{2}}^{2}+\bar{y}_{l_{1}}^{2}\right)$ for any $\bar{x}, \bar{y} \in \mathbb{R}^{d}$ and every $l_{1}, l_{2} \in\{1, \ldots, d\}$, by Young's inequality.

For the fourth term in (4.11) another decomposition of the integral and integration by parts yield that

$$
\begin{aligned}
& \int_{r}^{t_{j, n}} \partial_{x} \bar{B}_{k, l}\left(t_{j, n}, \underline{s}_{n},{ }_{n} Y\right) B_{H}\left(\underline{s}_{n}, \underline{s}_{n},{ }_{n} Y\right)\left(h(s)-h\left(s_{n}\right)\right) d_{n} W_{s}^{(l)} \\
& =\int_{r}^{t_{j, n}} \partial_{x} \bar{B}_{k, l}\left(s, \underline{s}_{n},{ }_{n} Y\right) B_{H}\left(\underline{s}_{n}, \underline{s}_{n},{ }_{n} Y\right) \Delta W_{s_{n}}^{(l)} \frac{\left(\bar{s}_{n}-s\right)}{\Delta \bar{s}_{n}} d h(s) \\
& \quad+\int_{r}^{t_{j, n}} \int_{r}^{t} \partial_{t} \partial_{x} \bar{B}_{k, l}\left(t, \underline{s}_{n},{ }_{n} Y\right) B_{H}\left(\underline{s}_{n}, \underline{s}_{n},{ }_{n} Y\right) \Delta W_{s_{n}}^{(l)} \frac{\left(\bar{s}_{n}-s\right)}{\Delta \bar{s}_{n}} d h(s) d t
\end{aligned}
$$

for each $j \in\left\{1, \ldots, k_{n}\right\}$, any $k \in\{1, \ldots, m\}$ and every $l \in\{1, \ldots, d\}$. Hence, from the Cauchy-Schwarz inequality we get that

$$
\begin{aligned}
& E\left[\max _{j \in\left\{0, \ldots, k_{n}\right\}} \sum_{k=1}^{m}\left|\sum_{l=1}^{d} \int_{r}^{t_{j, n}} \partial_{x} \bar{B}_{k, l}\left(t_{j, n}, \underline{s}_{n},{ }_{n} Y\right) B_{H}\left(\underline{s}_{n}, \underline{s}_{n},{ }_{n} Y\right)\left(h(s)-h\left(s_{n}\right)\right) d_{n} W_{s}^{(l)}\right|^{2}\right] \\
& \leq 2\|h\|_{1,2, r}^{2} \int_{r}^{T} \sum_{k=1}^{m} \sum_{l=1}^{d} E\left[\left|\partial_{x} \bar{B}_{k, l}\left(s, \underline{s}_{n},{ }_{n} Y\right) B_{H}\left(\underline{s}_{n}, \underline{s}_{n},{ }_{n} Y\right)\right|^{2}\right] E\left[\left|\Delta W_{s_{n}}^{(l)}\right|^{2}\right] d s \\
& \quad+2\|h\|_{1,2, r}^{2}(T-r) \int_{r}^{T} \int_{r}^{t} \sum_{k=1}^{m} E\left[\left|\sum_{l=1}^{d} \partial_{t} \partial_{x} \bar{B}_{k, l}\left(t, \underline{s}_{n},{ }_{n} Y\right) B_{H}\left(\underline{s}_{n}, \underline{s}_{n},{ }_{n} Y\right) \Delta W_{s_{n}}^{(l)}\right|^{2}\right] d s d t \\
& \leq c_{2,4}\left|\mathbb{T}_{n}\right|\left(1+E\left[\left\|_{n} Y^{r}\right\|_{\infty}^{2}\right]\right)^{\kappa}
\end{aligned}
$$

with $c_{2,4}:=2^{3}\left(1+(T-r)^{2} / 2\right)(T-r)\|h\|_{1,2, r}^{2} c^{2} \bar{c}^{2}\left(1+\underline{c}_{2}\right)^{\kappa}$, because $\Delta W_{s_{n}}^{(1)}, \ldots, \Delta W_{s_{n}}^{(d)}$ are pairwise independent and independent of $\mathscr{F}_{\underline{s}_{n}}$ for all $s \in[r, T]$.

The fifth expression in (4.11) can be treated in a similar way as the third. Namely, we set ${ }_{l, n} U_{s}:=\left({ }_{n} W_{s}-{ }_{n} W_{s_{n}}\right)_{n} \dot{W}_{s}^{(l)}-(1 / 2) \gamma_{n}(s) e_{l}$ for every $s \in[r, T]$ and rewrite that

$$
\begin{aligned}
& \int_{r}^{t_{j, n}} \partial_{x} \bar{B}_{k, l}\left(t_{j, n}, \underline{s}_{n},{ }_{n} Y\right) \bar{B}\left(\underline{s}_{n}, \underline{s}_{n},{ }_{n} Y\right)_{l, n} U_{s} d s \\
& =\frac{1}{2} \sum_{i=1}^{j-1} \partial_{x} \bar{B}_{k, l}\left(t_{i, n}, t_{i-1, n},{ }_{n} Y\right) \bar{B}\left(t_{i-1, n}, t_{i-1, n},{ }_{n} Y\right)_{l, n} V_{i} \\
& \quad+\frac{1}{2} \int_{t_{1, n}}^{t_{j, n}} \sum_{i=1}^{i_{n}(t)} \partial_{t} \partial_{x} \bar{B}_{k, l}\left(t, t_{i-1, n},{ }_{n} Y\right) \bar{B}\left(t_{i-1, n}, t_{i-1, n},{ }_{n} Y\right)_{l, n} V_{i} d t
\end{aligned}
$$

for all $j \in\left\{1, \ldots, k_{n}\right\}$, each $k \in\{1, \ldots, m\}$ and every $l \in\{1, \ldots, d\}$. Thus, from the estimate (4.10) we can again infer that

$$
\begin{aligned}
& E\left[\max _{j \in\left\{0, \ldots, k_{n}\right\}} \sum_{k=1}^{m}\left|\int_{r}^{t_{j, n}} \sum_{l=1}^{d} \partial_{x} \bar{B}_{k, l}\left(t_{j, n}, \underline{s}_{n},{ }_{n} Y\right) \bar{B}\left(\underline{s}_{n}, \underline{s}_{n},{ }_{n} Y\right)_{l, n} U_{s} d s\right|^{2}\right] \\
& \leq 2^{2}\left|\mathbb{T}_{n}\right| \sum_{i=1}^{k_{n}-1} \Delta t_{i, n} \sum_{k=1}^{m} \sum_{l=1}^{d} E\left[\left|\partial_{x} \bar{B}_{k, l}\left(t_{i, n}, t_{i-1, n},{ }_{n} Y\right) \bar{B}\left(t_{i-1, n}, t_{i-1, n},{ }_{n} Y\right)\right|^{2}\right]
\end{aligned}
$$


Support characterization for stochastic Volterra integral equations

$$
\begin{aligned}
& +2^{-1}(T-r) \int_{t_{1, n}}^{T} \sum_{k=1}^{m} E\left[\left|\sum_{i=1}^{i_{n}(t)} \sum_{l=1}^{d} \partial_{t} \partial_{x} \bar{B}_{k, l}\left(t, t_{i-1, n},{ }_{n} Y\right) \bar{B}\left(t_{i-1, n}, t_{i-1, n},{ }_{n} Y\right)_{l, n} V_{i}\right|^{2}\right] d t \\
\leq & c_{2,5}\left|\mathbb{T}_{n}\right|
\end{aligned}
$$

for $c_{2,5}:=2^{2}\left(1+(T-r)^{2}\right)(T-r) c^{2} \bar{c}^{2}$. For the sixth expression in (4.11) we decompose the integral and apply Itô's formula to the effect that

$$
\begin{aligned}
& \int_{r}^{t_{j, n}} \partial_{x} \bar{B}_{k, l}\left(t_{j, n}, \underline{s}_{n},{ }_{n} Y\right) \Sigma\left(\underline{s}_{n}, \underline{s}_{n},{ }_{n} Y\right)\left(W_{s}-W_{s_{n}}\right) d_{n} W_{s}^{(l)} \\
& =\int_{r}^{t_{j, n}} \partial_{x} \bar{B}_{k, l}\left(s, \underline{s}_{n},{ }_{n} Y\right) \Sigma\left(\underline{s}_{n}, \underline{s}_{n},{ }_{n} Y\right) \frac{\left(\bar{s}_{n}-s\right)}{\Delta \bar{s}_{n}} \Delta W_{s_{n}}^{(l)} d W_{s} \\
& \quad+\int_{r}^{t_{j, n}} \int_{r}^{t} \partial_{t} \partial_{x} \bar{B}_{k, l}\left(t, \underline{s}_{n},{ }_{n} Y\right) \Sigma\left(\underline{s}_{n}, \underline{s}_{n},{ }_{n} Y\right) \frac{\left(\bar{s}_{n}-s\right)}{\Delta \bar{s}_{n}} \Delta W_{s_{n}}^{(l)} d W_{s} d t \quad \text { a.s. }
\end{aligned}
$$

for all $j \in\left\{1, \ldots, k_{n}\right\}$, each $k \in\{1, \ldots, m\}$ and every $l \in\{1, \ldots, d\}$. Hence, by utilizing that $\Delta W_{s_{n}}^{(1)}, \ldots, \Delta W_{s_{n}}^{(d)}$ are pairwise independent for any $s \in[r, T]$, we estimate that

$$
\begin{aligned}
& E\left[\max _{j \in\left\{0, \ldots, k_{n}\right\}} \sum_{k=1}^{m}\left|\sum_{l=1}^{d} \int_{r}^{t_{j, n}} \partial_{x} \bar{B}_{k, l}\left(t_{j, n}, \underline{s}_{n},{ }_{n} Y\right) \Sigma\left(\underline{s}_{n}, \underline{s}_{n},{ }_{n} Y\right)\left(W_{s}-W_{s_{n}}\right) d_{n} W_{s}^{(l)}\right|^{2}\right] \\
& \leq 2 w_{2}\left|\mathbb{T}_{n}\right| \int_{r}^{T} \sum_{k=1}^{m} \sum_{l=1}^{d} E\left[\left|\partial_{x} \bar{B}_{k, l}\left(s, \underline{s}_{n},{ }_{n} Y\right) \Sigma\left(\underline{s}_{n}, \underline{s}_{n},{ }_{n} Y\right)\right|^{2}\right] d s \\
& \quad+2 w_{2}(T-r) \int_{r}^{T} \int_{r}^{t} \sum_{k=1}^{m} \sum_{l=1}^{d} E\left[\left|\partial_{t} \partial_{x} \bar{B}_{k, l}\left(t, \underline{s}_{n},{ }_{n} Y\right) \Sigma\left(\underline{s}_{n}, \underline{s}_{n},{ }_{n} Y\right)\right|^{2}\right] \Delta s_{n} d s d t \leq c_{2,6}\left|\mathbb{T}_{n}\right|,
\end{aligned}
$$

where $c_{2,6}:=2 w_{2}\left(1+(T-r)^{2} / 2\right)(T-r) c^{2} \bar{c}^{2}$.

Finally, for the seventh term in (4.11) we define an $\mathbb{R}^{m}$-valued $\mathscr{F}_{t_{i-1, n}}$-measurable random vector by

$$
{ }_{n} X_{i}:=\frac{1}{\Delta t_{i+1, n}} \int_{t_{i, n}}^{t_{i+1, n}} \int_{t_{i-1, n}}^{s} \int_{r}^{t_{i-1, n}} \partial_{v} \bar{B}\left(v, u,{ }_{n} Y\right) d_{n} W_{u} d v d s,
$$

which satisfies $E\left[\left.\left.\right|_{n} X_{i}\right|^{2}\right] \leq 2^{2} \hat{w}_{2,1}(T-r)^{2} c^{2}\left|\mathbb{T}_{n}\right|$ for any $i \in\left\{1, \ldots, k_{n}-1\right\}$. Then we have

$$
\begin{aligned}
& \int_{r}^{t_{j, n}} \partial_{x} \bar{B}_{k, l}\left(t_{j, n}, \underline{s}_{n},{ }_{n} Y\right) \int_{\underline{s}_{n}}^{s} \int_{r}^{\underline{s}_{n}} \partial_{v} \bar{B}\left(v, u,{ }_{n} Y\right) d_{n} W_{u} d v d_{n} W_{s}^{(l)} \\
& =\sum_{i=1}^{j-1} \partial_{x} \bar{B}_{k, l}\left(t_{i, n}, t_{i-1, n},{ }_{n} Y\right)_{n} X_{i} \Delta W_{t_{i, n}}^{(l)}+\int_{t_{1, n}}^{t_{j, n}} \sum_{i=1}^{i_{n}(t)} \partial_{t} \partial_{x} \bar{B}_{k, l}\left(t, t_{i-1, n},{ }_{n} Y\right)_{n} X_{i} \Delta W_{t_{i, n}}^{(l)} d t
\end{aligned}
$$

for all $j \in\left\{1, \ldots, k_{n}\right\}$, each $k \in\{1, \ldots, m\}$ and every $l \in\{1, \ldots, d\}$. As $\Delta W_{t_{i, n}}^{(1)}, \ldots, W_{t_{i, n}}^{(d)}$ are pairwise independent and independent of $\mathscr{F}_{t_{i-1, n}}$ for every $i \in\left\{1, \ldots, k_{n}\right\}$, it follows that

$$
\begin{aligned}
& E\left[\max _{j \in\left\{0, \ldots, k_{n}\right\}} \sum_{k=1}^{m}\left|\sum_{l=1}^{d} \int_{r}^{t_{j, n}} \partial_{x} \bar{B}_{k, l}\left(t_{j, n}, \underline{s}_{n},{ }_{n} Y\right) \int_{\underline{s}_{n}}^{s} \int_{r}^{\underline{s}_{n}} \partial_{v} \bar{B}\left(v, u,{ }_{n} Y\right) d_{n} W_{u} d v d_{n} W_{s}^{(l)}\right|^{2}\right] \\
& \leq 2^{3} \sum_{i=1}^{k_{n-1}} \Delta t_{i, n} \sum_{k=1}^{m} \sum_{l=1}^{d} E\left[\left|\partial_{x} \bar{B}_{k, l}\left(t_{i, n}, t_{i-1, n},{ }_{n} Y\right)_{n} X_{i}\right|^{2}\right] \\
& \quad+2(T-r) \int_{t_{1, n}}^{T} \sum_{k=1}^{m} E\left[\left|\sum_{i=1}^{i_{n}(t)} \sum_{l=1}^{d} \partial_{t} \partial_{x} \bar{B}_{k, l}\left(t, t_{i-1, n},{ }_{n} Y\right)_{n} X_{i} \Delta W_{t_{i, n}}^{(l)}\right|^{2}\right] d t \leq c_{2,7}\left|\mathbb{T}_{n}\right|
\end{aligned}
$$

with $c_{2,7}:=2^{5} \hat{w}_{2,1}\left(1+(T-r)^{2}\right)(T-r)^{3} c^{2} \bar{c}^{2}$, by virtue of the estimate (4.10). Hence, we complete the proof by setting $c_{2}:=7\left(c_{2,1}+\cdots+c_{2,7}\right)$. 


\section{Proofs of the convergence result and the support theorem}

\subsection{Proofs of Lemmas 2.2 and 1.1}

Proof of Lemma 2.2. (i) If $\Sigma$ vanishes, then (2.6) can be solved in a pathwise sense. Namely, for any $x \in C\left([0, T], \mathbb{R}^{m}\right)$ and each $w \in C\left([0, T], \mathbb{R}^{d}\right)$ Proposition 3 in [7] yields a unique solution $y_{x, w} \in W_{r}^{1,2}\left([0, T], \mathbb{R}^{m}\right)$ to the Volterra integral equation

$$
y_{x, w}(t)=x(r)+\int_{r}^{t} \underline{B}\left(t, s, y_{x, w}\right)+B_{H}\left(t, s, y_{x, w}\right) \dot{h}(s)+\bar{B}\left(t, s, y_{x, w}\right) \dot{L}_{n}(w)(s) d s
$$

for $t \in[r, T]$ with $y_{x, w}^{r}=x^{r}$, by the absolute continuity condition on $\underline{B}, B_{H}$ and $\bar{B}$ in (C.5). Thus, pathwise uniqueness for (2.6) holds and the process ${ }_{n} Y \in \mathscr{C}\left([0, T], \mathbb{R}^{m}\right)$ given by ${ }_{n} Y_{t}:=y_{n} \xi, W(t)$ is the unique strong solution with ${ }_{n} Y^{r}={ }_{n} \xi^{r}$ a.s., since the map $C\left([0, T], \mathbb{R}^{m}\right) \times C\left([0, T], \mathbb{R}^{d}\right) \rightarrow W_{r}^{1,2}\left([0, T], \mathbb{R}^{m}\right),(x, w) \mapsto y_{x, w}$ is Borel measurable.

Now suppose that $\bar{B}=\bar{b} \Sigma$ for some measurable function $\bar{b}:[r, T] \rightarrow \mathbb{R}$ such that $\int_{r}^{T} \bar{b}(s)^{2} d s<\infty$. In this case, according to Lemma 35 in [7], we may introduce a martingale ${ }_{n} \bar{Z} \in \mathscr{C}([0, T], \mathbb{R})$ by ${ }_{n} \bar{Z}^{r}=1$ and

$$
{ }_{n} \bar{Z}_{t}=\exp \left(-\int_{r}^{t} \bar{b}(s)_{n} \dot{W}_{s}^{\prime} d W_{s}-\frac{1}{2} \int_{r}^{t}\left|\bar{b}(s)_{n} \dot{W}_{s}\right|^{2} d s\right)
$$

for all $t \in[r, T]$ a.s. Then ${ }_{n} \bar{W} \in \mathscr{C}\left([0, T], \mathbb{R}^{d}\right)$ defined via ${ }_{n} \bar{W}_{t}:=W_{t}+\int_{r}^{r \vee t} \bar{b}(s) d_{n} W_{s}$ is a $d$-dimensional $\left(\mathscr{F}_{t}\right)_{t \in[0, T]}$-Brownian motion under the probability measure $\bar{P}_{n}$ on $(\Omega, \mathscr{F})$ given by $\bar{P}_{n}(A):=E\left[{ }_{n} \bar{Z}_{T} \mathbb{1}_{A}\right]$, by Girsanov's theorem.

We observe that a process $Y \in \mathscr{C}\left([0, T], \mathbb{R}^{m}\right)$ is a solution to (2.6) under $P$ if and only if it solves the path-dependent stochastic Volterra integral equation

$$
Y_{t}=Y_{r}+\int_{r}^{t} \underline{B}(t, s, Y)+B_{H}(t, s, Y) \dot{h}(s) d s+\int_{r}^{t} \Sigma(t, s, Y) d_{n} \bar{W}_{s}
$$

a.s. for $t \in[r, T]$ under $\bar{P}_{n}$. In the one-dimensional case $m=1$ pathwise uniqueness and strong existence for (5.1) can essentially be inferred from Theorem 4.3 in [13] when considering the drift $\underline{B}+B_{H} \dot{h}$ and the diffusion $\Sigma$. In the general case, pathwise uniqueness follows, for instance, by proceeding similarly as in the proof of Lemma 27 in [7], due to the absolute continuity condition on $\underline{B}, B_{H}$ and $\Sigma$.

For strong existence we may assume that $\bar{b}=0$ and recall from Proposition 11 in [7] that the linear space $\mathscr{C}^{p_{n}}\left([0, T], \mathbb{R}^{m}\right)$ of all $X \in \mathscr{C}\left([0, T], \mathbb{R}^{m}\right)$ for which $E\left[\|X\|_{\infty}^{p_{n}}\right]$ is finite, endowed with the seminorm $\mathscr{C}^{p_{n}}\left([0, T], \mathbb{R}^{m}\right) \rightarrow \mathbb{R}_{+}, X \mapsto E\left[\|X\|_{\infty}^{p_{n}}\right]^{1 / p_{n}}$, is complete. Then the operator $\Psi: \mathscr{C}^{p_{n}}\left([0, T], \mathbb{R}^{m}\right) \rightarrow \mathscr{C}\left([0, T], \mathbb{R}^{m}\right)$ specified by requiring that

$$
\begin{aligned}
\Psi(Y)_{t}= & { }_{n} \xi_{r \wedge t}+\int_{r}^{r \vee t} \underline{B}(s, s, Y)+B_{H}(s, s, Y) \dot{h}(s) d s+\int_{r}^{r \vee t} \Sigma(s, s, Y) d W_{s} \\
& +\int_{r}^{r \vee t} \int_{r}^{v} \partial_{v} \underline{B}(v, u, Y)+\partial_{v} B_{H}(v, u, Y) \dot{h}(u) d u+\int_{r}^{v} \partial_{v} \Sigma(v, u, Y) d W_{u} d v
\end{aligned}
$$

for any $t \in[0, T]$ a.s. maps $\mathscr{C}^{p_{n}}\left([0, T], \mathbb{R}^{m}\right)$ into itself and satisfies $E\left[\left\|\Psi(Y)^{t}-\Psi(\tilde{Y})^{t}\right\|_{\infty}^{p_{n}}\right]$ $\leq c_{p_{n}} \int_{r}^{t} E\left[\left\|Y^{s}-\tilde{Y}^{s}\right\|_{\infty}^{p_{n}}\right] d s$ for any $Y, \tilde{Y} \in \mathscr{C}^{p_{n}}\left([0, T], \mathbb{R}^{m}\right)$ and all $t \in[r, T]$, where we have set $c_{p_{n}}:=3^{p_{n}}(1+T-r)^{3 p_{n} / 2}\left((T-r)^{p_{n} / 2}+\|h\|_{1,2, r}^{p_{n}}+w_{p_{n}}\right) \lambda^{p_{n}}$.

Hence, it follows as in the proof of Proposition 5 in [7] that the sequence $(k, n=)_{k \in \mathbb{N}_{0}}$ in $\mathscr{C}^{p_{n}}\left([0, T], \mathbb{R}^{m}\right)$, recursively defined by ${ }_{0, n} Y:={ }_{n} \xi$ and ${ }_{k, n} Y:=\Psi\left({ }_{k-1, n} Y\right)$ for all $k \in \mathbb{N}$, converges to the a.s. unique fixed-point ${ }_{n} Y$ of $\Psi$, which is a solution to (5.1) and satisfies ${ }_{n} Y^{r}={ }_{n} \xi^{r}$ a.s. 
Regarding the claimed estimate, we let $p>2$ and $\alpha \in[0,1 / 2-1 / p)$. Then from Proposition 4.1 we obtain $c_{p}>0$ such that (4.1) holds and the Kolmogorov-Chentsov estimate (3.1) implies that

$$
E\left[\left(\left\|_{n} Y\right\|_{\alpha, r}-\left\|_{n} \xi^{r}\right\|_{\infty}\right)^{p}\right] \leq k_{\alpha, p, \frac{p}{2}-1} c_{p}(T-r)^{p\left(\frac{1}{2}-\alpha\right)}\left(1+E\left[\left\|_{n} \xi^{r}\right\|_{\infty}^{p}\right]\right)
$$

for every $n \in \mathbb{N}$. Hence, we set $c_{\alpha, p}:=2^{p-1}\left(1+k_{\alpha, p, p / 2-1} c_{p}(T-r)^{p(1 / 2-\alpha)}\right)$, then the triangle inequality gives the desired result.

(ii) Pathwise uniqueness, strong existence and the asserted bound can be directly inferred from (i) by replacing $\underline{B}$ by $\underline{B}+R, \bar{B}$ by 0 and $\Sigma$ by $\bar{B}+\Sigma$, since in this case the required condition (C.9) holds for the choice $\bar{b}=0$.

Proof of Lemma 1.1. (i) Pathwise uniqueness, the existence of a unique strong solution and the integrability condition follow from assertion (ii) of Lemma 2.2 by letting $\underline{B}=b$, $B_{H}=\bar{B}=0, \Sigma=\sigma$ and $\xi=\hat{x}$.

(ii) For $h \in W_{r}^{1, p}\left([0, T], \mathbb{R}^{d}\right)$ we set $F_{h}:=b-(1 / 2) \bar{\rho}+\sigma \dot{h}$, where $\bar{\rho}(t, s, x)=\rho(t, s, x)$ for any $s, t \in[r, T]$ with $s<t$ and all $x \in C\left([0, T], \mathbb{R}^{m}\right)$, as defined in Section 2.2.

First, since $\partial_{x} \sigma(\cdot, s, x)$ is absolutely continuous on $(s, T]$, so is $\rho(\cdot, s, x)$ and hence, $F_{h}(\cdot, s, x)$ for any $(s, x) \in[r, T) \times C\left([0, T], \mathbb{R}^{m}\right)$. Secondly, there are $c_{0}, \lambda_{0} \geq 0$ such that $\max \left\{|\sigma|,\left|\partial_{t} \sigma\right|,|\rho|,\left|\partial_{t} \rho\right|\right\} \leq c_{0}$ and

$$
|\bar{\rho}(s, s, x)-\bar{\rho}(s, s, y)|+\left|\partial_{t} \rho(t, s, x)-\partial_{t} \rho(t, s, y)\right| \leq \lambda_{0}\|x-y\|_{\infty}
$$

for all $s, t \in(r, T)$ with $s<t$ and every $x, y \in C\left([0, T], \mathbb{R}^{m}\right)$. These conditions ensure that the map $F_{h}$ satisfies $\left|F_{h}(s, s, x)\right|+\left|\partial_{t} F_{h}(t, s, x)\right| \leq c_{1}(1+|\dot{h}(s)|)\left(1+\|x\|_{\infty}\right)$ and

$$
\left|F_{h}(s, s, x)-F_{h}(s, s, y)\right|+\left|\partial_{t} F_{h}(t, s, x)-\partial_{t} F_{h}(t, s, y)\right| \leq \lambda_{1}(1+|\dot{h}(s)|)\|x-y\|_{\infty}
$$

for any $s, t \in(r, T)$ with $s<t$ and all $x, y \in C\left([0, T], \mathbb{R}^{m}\right)$, where $c_{1}:=3 \max \left\{c_{0}, c\right\}$ and $\lambda_{1}:=2 \max \left\{\lambda_{0}, \lambda\right\}$. As these are all the necessary assumptions, we invoke Proposition 3 in [7] to get a unique solution $x_{h}$ to (1.5), which satisfies $x_{h} \in W_{r}^{1, p}\left([0, T], \mathbb{R}^{m}\right)$.

To show the second assertion, we also let $g \in W_{r}^{1, p}\left([0, T], \mathbb{R}^{d}\right)$. Then for the constant $c_{p, 1}:=2^{2 p-2}(1+T-r)^{p} \max \{1, T-r\}^{p-1} \max \left\{c_{0}^{p}, \lambda_{1}^{p}\right\}$ we have

$$
\left\|x_{g}^{t}-x_{h}^{t}\right\|_{1, p, r}^{p} \leq c_{p, 1} \int_{r}^{t}|\dot{g}(s)-\dot{h}(s)|^{p}+\left(1+|\dot{h}(s)|^{p}\right)\left\|x_{g}^{s}-x_{h}^{s}\right\|_{1, p, r}^{p} d s
$$

for each $t \in[r, T]$, since $\|y\|_{\infty} \leq \max \{1, T-r\}^{1-1 / p}\|y\|_{1, p, r}$ for any $y \in W_{r}^{1, p}\left([0, T], \mathbb{R}^{m}\right)$. Hence, Gronwall's inequality gives $\left\|x_{g}-x_{h}\right\|_{1, p, r}^{p} \leq c_{p} \exp \left(c_{p}\|h\|_{1, p, r}^{p}\right)\|g-h\|_{1, p, r}^{p}$, where we have defined $c_{p}:=c_{p, 1} \exp \left((T-r) c_{p, 1}\right)$.

\subsection{Proofs of Theorems 2.3 and 1.2}

Proof of Theorem 2.3. By Lemma 3.1, which is applicable for $q=p / 2-1$ and $p_{0}=2$, due to Proposition 4.1 and Corollary 4.2, we merely have to show the first assertion, as the second follows from the first.

In this regard, we use the decomposition of Proposition 4.3 in second moment. Namely, it readily follows from the interpolation error estimate (3.2) that

$$
\lim _{n \uparrow \infty} E\left[\left\|L_{n}\left({ }_{n} Y\right)-{ }_{n} Y\right\|_{\infty}^{p}+\left\|L_{n}(Y)-Y\right\|_{\infty}^{p}\right] /\left|\mathbb{T}_{n}\right|^{\alpha p}=0 .
$$

Thus, Lemma 3.4, Proposition 3.5 and Hölder's inequality show that the claimed limit holds once we can justify that there is $c_{2}>0$ such that

$$
E\left[\max _{j \in\left\{0, \ldots, k_{n}\right\}}\left|\int_{r}^{t_{j, n}}\left(\bar{B}\left(t_{j, n}, s,{ }_{n} Y\right)-\bar{B}\left(t_{j, n}, \underline{s}_{n},{ }_{n} Y\right)\right)_{n} \dot{W}_{s}-R\left(t_{j, n}, \underline{s}_{n},{ }_{n} Y\right) \gamma_{n}(s) d s\right|^{2}\right]
$$


does not exceed $c_{2}\left|\mathbb{T}_{n}\right|^{1-2 / p}$ for each $n \in \mathbb{N}$. Based on the decomposition (4.7) and the hypothesis that $\partial_{x} \bar{B}$ is bounded, this fact follows from Proposition 4.4 and Lemma 4.5, in conjunction with Lemma 3.2 and Remark 3.3, and Proposition 4.6.

Proof of Theorem 1.2. First, we let $N_{\alpha}$ denote the $P$-null set of all $\omega \in \Omega$ such that $X(\omega) \notin C_{r}^{\alpha}\left([0, T], \mathbb{R}^{m}\right)$ and recall that the support of $P \circ X^{-1}$ in $C_{r}^{\alpha}\left([0, T], \mathbb{R}^{m}\right)$ coincides with the support of the inner regular probability measure

$$
\mathscr{B}\left(C_{r}^{\alpha}\left([0, T], \mathbb{R}^{m}\right)\right) \rightarrow[0,1], \quad B \mapsto P\left(\{X \in B\} \cap N_{\alpha}^{c}\right) .
$$

Then an application of Theorem 2.3 in the case that $\underline{B}=b-(1 / 2) \rho, B_{H}=0, \bar{B}=\sigma$, $\Sigma=0$ and $\xi_{n}=\xi=\hat{x}$ for all $n \in \mathbb{N}$ gives us (2.4), which in turn implies that the support of (5.2) is included in the closure of $\left\{x_{h} \mid h \in W_{r}^{1, p}\left([0, T], \mathbb{R}^{d}\right)\right\}$ relative to $\|\cdot\|_{\alpha, r}$.

Now we let $h \in W_{r}^{1, p}\left([0, T], \mathbb{R}^{d}\right)$ be fixed and recall from [7][Proof of Theorem 1] that for any $n \in \mathbb{N}$ and each $x \in C\left([0, T], \mathbb{R}^{d}\right)$ there is a unique solution $y_{h, n, x} \in C\left([0, T], \mathbb{R}^{d}\right)$ to the ordinary integral equation with running value condition

$$
y_{h, n, x}(t)=x(t)-\int_{r}^{r \vee t} \dot{h}(s)-\dot{L}_{n}\left(y_{h, n, x}\right)(s) d s \quad \text { for } t \in[0, T] .
$$

As the map $C\left([0, T], \mathbb{R}^{d}\right) \rightarrow C\left([0, T], \mathbb{R}^{d}\right), x \mapsto y_{h, n, x}$ is Lipschitz continuous on bounded sets, we may let ${ }_{h, n} W \in \mathscr{C}\left([0, T], \mathbb{R}^{d}\right)$ be given by ${ }_{h, n} W_{t}:=y_{h, n, W}(t)$ and introduce a martingale ${ }_{h, n} Z \in \mathscr{C}([0, T], \mathbb{R})$ by requiring that ${ }_{h, n} Z^{r}=1$ and

$$
{ }_{h, n} Z_{t}=\exp \left(\int_{r}^{t} \dot{h}(s)^{\prime}-\dot{L}_{n}(h, n W)(s)^{\prime} d W_{s}-\frac{1}{2} \int_{r}^{t}\left|\dot{h}(s)-\dot{L}_{n}(h, n W)(s)\right|^{2} d s\right)
$$

for any $t \in[r, T]$ a.s. By Girsanov's theorem, ${ }_{h, n} W$ is a $d$-dimensional $\left(\mathscr{F}_{t}\right)_{t \in[0, T]}$-Brownian motion under the probability measure $P_{h, n}$ on $(\Omega, \mathscr{F})$ given by $P_{h, n}(A):=E\left[{ }_{h, n} Z_{T} \mathbb{1}_{A}\right]$ and $X$ is a strong solution to the stochastic Volterra integral equation

$$
X_{t}=X_{r}+\int_{r}^{t} b(t, s, X)+\sigma(t, s, X)\left(\dot{h}-\dot{L}_{n}(h, n W)\right)(s) d s+\int_{r}^{t} \sigma(t, s, X) d_{h, n} W_{s}
$$

a.s. for $t \in[r, T]$ under $P_{h, n}$. Hence, let ${ }_{n} Y$ be the unique strong solution to (2.6) when $\underline{B}=b, B_{H}=\sigma, \bar{B}=-\sigma$ and $\Sigma=\sigma$ with ${ }_{n} Y^{r}=\hat{x}^{r}$ a.s., then uniqueness in law implies that $P\left(\left\|_{n} Y-x_{h}\right\|_{\alpha, r} \geq \varepsilon\right)=P_{h, n}\left(\left\|X-x_{h}\right\|_{\alpha, r} \geq \varepsilon\right)$ for any $\varepsilon>0$. This shows that Theorem 2.3 also yields (2.5) and the claimed representation follows.

\section{References}

[1] Aida, S.: Support theorem for diffusion processes on Hilbert spaces. Publ. Res. Inst. Math. Sci. 26, (1990), no. 6, 947-965. MR-1079903

[2] Bally, V., Caramellino, L. and Cont, R.: Stochastic integration by parts and functional Itô calculus. Advanced Courses in Mathematics. CRM Barcelona, Birkhäuser/Springer, [Cham], 2016, Lecture notes of the Barcelona Summer School on Stochastic Analysis held in Barcelona, July 23-27, 2012, Edited by Frederic Utzet and Josep Vives. MR-3381599

[3] Bally, V., Millet, A. and Sanz-Solé, M.: Approximation and support theorem in Hölder norm for parabolic stochastic partial differential equations. Ann. Probab. 23, (1995), no. 1, 178-222. MR-1330767

[4] Ben Arous, G., Grădinaru, M. and Ledoux, M.: Hölder norms and the support theorem for diffusions. Ann. Inst. H. Poincaré Probab. Statist. 30, (1994), no. 3, 415-436. MR-1288358

[5] Cont, R. and Fournie, D.: A functional extension of the Ito formula. C. R. Math. Acad. Sci. Paris, 348, (2010), no. 1-2, 57-61. MR-2586744 
[6] Cont, R. and Fournié, D.-A.: Functional Itô calculus and stochastic integral representation of martingales. Ann. Probab. 41, (2013), no. 1, 109-133. MR-3059194

[7] Cont, R. and Kalinin, A.: On the support of solutions to stochastic differential equations with path-dependent coefficients. Stochastic Process. Appl. 130, (2020), no. 5, 2639-2674. MR-4080725

[8] Dupire, B.: Functional Itô calculus. Quant. Finance 19, (2019), no. 5, 721-729. MR-3939653

[9] Gyöngy, I. and Pröhle, T.: On the approximation of stochastic differential equation and on Stroock-Varadhan's support theorem. Comput. Math. Appl. 19, (1990), no. 1, 65-70. MR-1026782

[10] Mao, X.: Stochastic Differential Equations and Applications. Second ed., Horwood Publishing Limited, Chichester, 2008. MR-2380366

[11] Millet, A. and Sanz-Solé, M.: A simple proof of the support theorem for diffusion processes. Séminaire de Probabilités, XXVIII, Lecture Notes in Math., vol. 1583, Springer, Berlin, 1994, pp. 36-48. MR-1329099

[12] Pakkanen, M. S.: Stochastic integrals and conditional full support. J. Appl. Probab. 47, (2010), no. 3, 650-667. MR-2731340

[13] Protter, P.: Volterra equations driven by semimartingales. Ann. Probab. 13, (1985), no. 2, 519-530. MR-781420

[14] Stroock, D. W. and Varadhan, S. R. S.: On the support of diffusion processes with applications to the strong maximum principle. Proceedings of the Sixth Berkeley Symposium on Mathematical Statistics and Probability (Univ. California, Berkeley, Calif., 1970/1971), Vol. III: Probability theory, 1972, pp. 333-359. MR-0400425

[15] Veraar, M.: The stochastic Fubini theorem revisited. Stochastics 84, (2012), no. 4, 543-551. MR-2966093

Acknowledgments. The author is grateful for the support from Imperial College London by a Chapman fellowship. 


\section{Electronic Journal of Probability Electronic Communications in Probability}

\section{Advantages of publishing in EJP-ECP}

- Very high standards

- Free for authors, free for readers

- Quick publication (no backlog)

- Secure publication $\left(\mathrm{LOCKSS}^{1}\right)$

- Easy interface (EJMS²)

\section{Economical model of EJP-ECP}

- Non profit, sponsored by $\mathrm{IMS}^{3}, \mathrm{BS}^{4}$, ProjectEuclid ${ }^{5}$

- Purely electronic

\section{Help keep the journal free and vigorous}

- Donate to the IMS open access fund ${ }^{6}$ (click here to donate!)

- Submit your best articles to EJP-ECP

- Choose EJP-ECP over for-profit journals

\footnotetext{
${ }^{1}$ LOCKSS: Lots of Copies Keep Stuff Safe http://www. lockss.org/

${ }^{2}$ EJMS: Electronic Journal Management System http://www.vtex.lt/en/ejms.html

${ }^{3}$ IMS: Institute of Mathematical Statistics http://www.imstat.org/

${ }^{4}$ BS: Bernoulli Society http://www. bernoulli-society.org/

${ }^{5}$ Project Euclid: https://projecteuclid.org/

${ }^{6}$ IMS Open Access Fund: http://www.imstat.org/publications/open.htm
} 